\title{
Phase stability and physical properties of nanolaminated materials from first principles
}

\author{
Andreas Thore
}

\section{U UNNKERNG}

\author{
Materials Design \\ Thin Film Physics Division \\ Department of Physics, Chemistry and Biology (IFM) \\ Linköping University \\ SE-581 83 Linköping, Sweden
}

Linköping 2016 
The cover shows an exponential curve. It is a visual representation of Moore's law Gordon Moore's observation that the number of components, such as transistors, per integrated circuit doubles roughly every two years. The exponential increase in computational power that this has entailed has made computational materials science possible. Curiously enough, computational materials science is now an important driver of this increase, and, consequently, a driver of its own capabilities.

ISBN 978-91-7685-835-6

ISSN 0345-7524

Printed by LiU-tryck, Linköping 2016 


\section{Abstract}

The MAX phase family is a set of nanolaminated, hexagonal materials typically comprised of three elements: a transition metal $(M)$, an A-group element $(A)$, and carbon and/or nitrogen $(X)$. In this thesis, first-principles based methods have been used to investigate the phase stability and physical properties of a number of MAX and MAX-like phases.

Most theoretical work on MAX phase stability use the constraint of $0 \mathrm{~K}$ conditions, due to the very high computational cost of including temperature dependent effects such as lattice vibrations and electronic excitations for all relevant competing phases in the ternary or multinary chemical space. Despite this, previous predictions of the existence of new MAX phases have to a large extent been experimentally verified. In an attempt to provide a possible explanation for this consistency, and thus help strengthen the confidence in future predictions, we have calculated the temperature dependent phase stability of $\mathrm{Ti}_{n+1} \mathrm{AlC}_{n}$, to date the most studied MAX phases. We show that both the electronic and vibrational contribution to the Gibbs free energies of the MAX phases are cancelled by the corresponding contributions to the Gibbs free energies of the competing phases. We further show that this is the case even when thermal expansion is considered.

We have also investigated the stability of two hypothetical MAX-like phases, $\mathrm{V}_{2} \mathrm{Ga}_{2} \mathrm{C}$ and $\left(\mathrm{Mo}_{1-x} \mathrm{~V}_{x}\right)_{2} \mathrm{Ga}_{2} \mathrm{C}$, motivated by a search for ways to attain new two-dimensional MAX phase derivatives, so-called MXenes. We predict that it is possible to synthesize both phases. For $x \leq 0.25$, stability of $\left(\mathrm{Mo}_{1-x} \mathrm{~V}_{x}\right)_{2} \mathrm{Ga}_{2} \mathrm{C}$ is indicated for both ordered and disordered solid solutions on the $M$ sublattice. For $x=0.5$ and $x \geq 0.75$, stability is only indicated for disordered solutions. The ordered solutions are stable at temperatures below $1000 \mathrm{~K}$, whereas stabilization of the disordered solutions requires temperatures of up to $2100 \mathrm{~K}$, depending on the $\mathrm{V}$ concentration.

Finally, we have investigated the electronic, vibrational, and magnetic properties of the recently synthesized $\mathrm{MAX}$ phase $\mathrm{Mn}_{2} \mathrm{GaC}$. We show that the electronic band structure is anisotropic, and determine the bulk, shear, and Young's modulus to be 157, 93, and $233 \mathrm{GPa}$, respectively, and Poisson's ratio to be 0.25 . We further predict the magnetic critical order-disorder temperature of $\mathrm{Mn}_{2} \mathrm{GaC}$ to be $660 \mathrm{~K}$. We base the predictions on Monte Carlo simulations of a bilinear Heisenberg Hamiltonian constructed from magnetic exchange interaction parameters derived using two 
different supercell methods: the novel magnetic direct cluster averaging method (MDCA), and the Connolly-Williams method (CW). We conclude that $\mathrm{CW}$ is less computationally expensive than MDCA for chemically and topologically ordered phases such as $\mathrm{Mn}_{2} \mathrm{GaC}$. 


\section{Populärvetenskaplig sammanfattning}

Materialvetenskap är ett område inom vilket ett mål är att framställa nya material med egenskaper som är till gagn för mänskligheten. Praktiskt taget all teknik, från stekpannor och golfklubbor till lysdioder och datorkomponenter, är på ett eller annat sätt åtminstone delvis resultat av materialvetenskapliga landvinningar.

Under nästan hela dess historia har materialvetenskaplig forskning uteslutande bedrivits genom fysikaliska experiment, men tack vare att framsteg inom den teoretiska fysiken lett till förenklade sätt att beräkna hur atomer interagerar med varandra, samt en snabb utveckling av datorers beräkningskapacitet, är det nu möjligt att även använda sig av datorsimuleringar. Med datorsimuleringar kan man på kort tid utföra ett stort antal virtuella experiment för att undersöka både möjligheten att framställa olika material och bestämma deras egenskaper, vilket innebär att mängden fysikaliska experiment, som ofta är betydligt mer tidskrävande, kan minskas.

Denna avhandling är helt och hållet baserad på datorsimuleringar, och den fokuserar främst på så kallade MAX-faser. En MAX-fas kännetecknas dels av att den har en lagrad kristallstruktur - atomernas positioner i materialet formar plan staplade ovanpå varandra - och dels av att den innehåller tre olika typer av grundämnen: övergångsmetaller ( $M$-atomer), A-gruppsatomer (främst från grupp 13 och $14 \mathrm{i}$ det periodiska systemet), samt kol och/eller kväve (X-atomer). MAX-faser har kommersiell potential på grund av att de kombinerar metalliska och keramiska egenskaper på ett fördelaktigt sätt.

I avhandlingen studeras varför det inte verkar spela någon roll att simuleringar av MAX-fasers stabilitet - en indikator på huruvida de är möjliga att framställa eller inte - väldigt sällan tar hänsyn till temperaturberoende effekter, det vill säga varför simuleringar hittills visat sig stämma väldigt bra överens med experimentella resultat trots att MAX-faser alltid skapas långt över rumstemperatur. Mina egna simuleringar av stabiliteten hos tre MAX-faser som innehåller titan, aluminum och kol, visar att detta förmodligen beror på att de temperaturberoende effekterna i MAX-faser och i konkurrerande faser tar ut varandra. Detta innebär att det nu med större säkerhet än tidigare går att lita på simuleringar av MAX-fasers stabilitet där temperatureffekter inte tagits med i beräkningarna, vilket är av stor praktiskt betydelse eftersom dessa effekter är förenade med väldigt mycket extra beräkningstid.

Vidare har jag räknat på möjligheten att framställa två MAX-fasliknande material: $\mathrm{V}_{2} \mathrm{Ga}_{2} \mathrm{C}$ och $\left(\mathrm{Mo}_{1-x} \mathrm{~V}_{x}\right)_{2} \mathrm{Ga}_{2} \mathrm{C}$, där det senare materialet innehåller en legering av grundämnena molybden och vanadin. Beräkningarna visar att det borde gå att 
framställa båda materialen, men att temperaturen som krävs för framställning av (Mo1$\left.{ }_{x} \mathrm{~V}_{x}\right)_{2} \mathrm{Ga}_{2} \mathrm{C}$ beror på mängdförhållandet mellan vanadin och molybden, och hur de två atomslagen är distribuerade i materialet.

Till sist har jag studerat egenskaperna hos $\mathrm{Mn}_{2} \mathrm{GaC}$, som är ett nytillskott till MAXfasfamiljen och en av de första magnetiska MAX-faserna. Jag har bland annat beräknat dess elastiska egenskaper, som ger ett mått på hur bra det är på att stå emot externa, mekaniska krafter, och vid vilken temperatur materialet går från att vara en permanent magnet till att bli paramagnetiskt. För det senare ändamålet har jag dessutom utvärderat två olika metoder för beräkning av interaktionsstyrkan mellan magnetiska atomer, varav en är relativt nyutvecklad och tidigare otestad på kemiskt och geometriskt välordnade material som $\mathrm{Mn}_{2} \mathrm{GaC}$. Slutsatsen är dels att denna nya metod är bättre lämpad för mer oordnade material, och dels att övergångstemperaturen ändå, trots att beräkningarna har en betydande felmarginal, sannolikt ligger långt över rumstemperatur. Detta ökar potentialen för $\mathrm{Mn}_{2} \mathrm{GaC}$ i olika tillämpningar där magnetismen utnyttjas. 


\section{Preface}

This thesis is the result of my work from August 2011 to February 2016 as a Ph.D. student within the Materials Design group of the Thin Film Physics Division at the Department of Physics, Chemistry, and Biology (IFM) at Linköping University. The research has been funded by the European Research Council under the European Community Seventh Framework Program (FP7/2007-2013)/ERC Grant agreement No. [258509]. 



\section{Acknowledgements}

During my years as a Ph.D. student, I have been fortunate to work very closely with three persons:

Johanna Rosén, my main supervisor

Björn Alling, my co-supervisor

Martin Dahlqvist, colleague and de facto co-supervisor

I am very grateful for your support and encouragement!

I am also very grateful to Peter Johansson, my former supervisor as an undergraduate student at Örebro University.

Finally, I would like to thank the rest of my colleagues at IFM for making it such a pleasant workplace. I have really enjoyed my time here. 



\section{Papers included in this thesis}

\section{Paper I}

Temperature dependent phase stability of nanolaminated ternaries from first-principles calculations

A. Thore, M. Dahlqvist, B. Alling, and J. Rosen, Computational Materials Science 91, 251 (2014).

My contribution: I participated in the planning of and carried out all calculations. I was responsible for writing the paper.

\section{Paper II}

Phase stability of the nanonlaminates $V_{2} G a_{2} C$ and $\left(M o_{1-\mathrm{x}} V_{\mathrm{x}}\right)_{2} \mathrm{Ga} a_{2} \mathrm{C}$ from first-principles calculations

A. Thore, M. Dahlqvist, B. Alling, and J. Rosen, submitted for publication

My contribution: I participated in the planning of and carried out some of the calculations. I was responsible for writing the paper.

\section{Paper III}

First-principles calculations of the electronic, vibrational, and elastic properties of the magnetic laminate $\mathrm{Mn}_{2} \mathrm{GaC}$

A. Thore, M. Dahlqvist, B. Alling, and J. Rosen, Journal of Applied Physics 116 (2014).

My contribution: I participated in the planning of and carried out most of the calculations. I was responsible for writing the paper.

\section{Paper IV}

Magnetic exchange interactions and critical temperature of the nanolaminate $M n_{2} \mathrm{GaC}$ from first-principles supercell methods

A. Thore, M. Dahlqvist, B. Alling, and J. Rosen, submitted for publication

My contribution: I participated in the planning of and carried out all calculations. I was responsible for writing the paper. 


\section{Related papers}

\section{Paper V}

Magnetically driven anisotropic structural changes in the atomic laminate $\mathrm{Mn}{ }_{2} \mathrm{GaC}$

M. Dahlqvist, A. S. Ingason, B. Alling, F. Magnus, A. Thore, A. Petruhins, A. Mockute, U. B. Arnalds, M. Sahlberg, B. Hjörvarsson, I. A. Abrikosov, and J. Rosen, Physical Review B 93, (2016). 


\section{Contents}

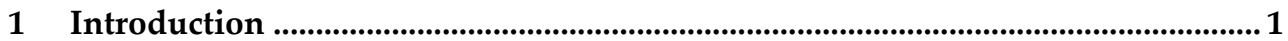

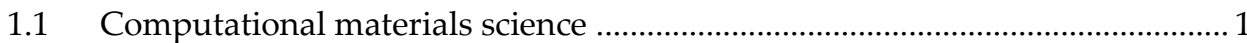

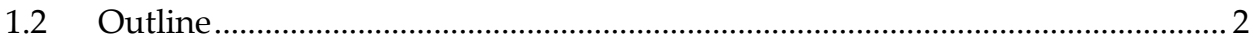

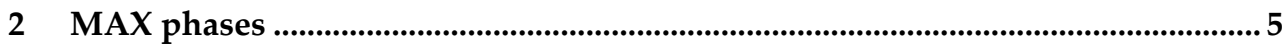

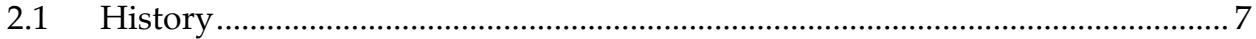

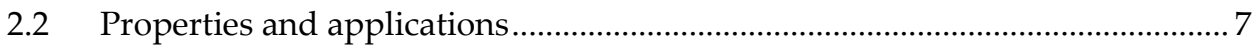

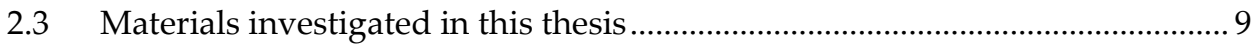

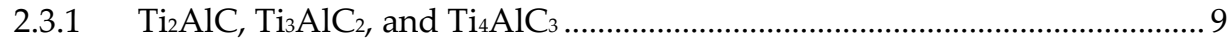

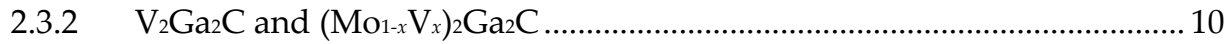

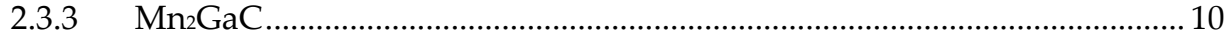

3 Density functional theory.......................................................................................... 11

3.1 The energy of a system of interacting particles .............................................. 11

3.2 The Hohenberg-Kohn Theorems ....................................................................... 12

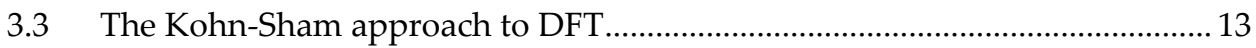

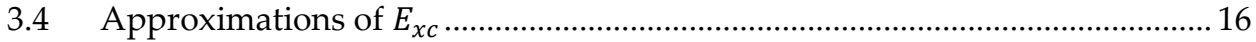

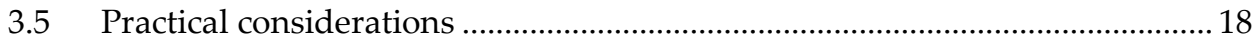

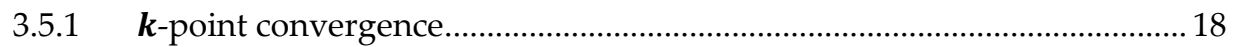

3.5.2 Energy cutoff convergence and pseudopotentials .................................... 20

4 Phase stability calculations from first principles ................................................... 23

4.1 Thermodynamic stability and metastability ................................................... 23

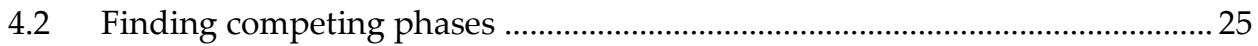

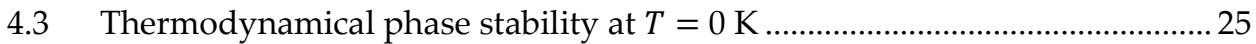

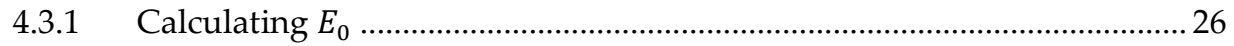

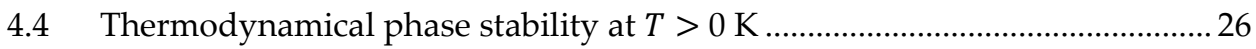

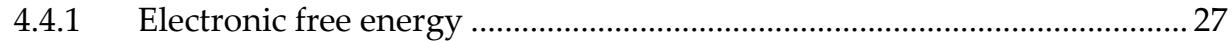

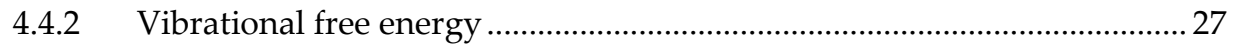

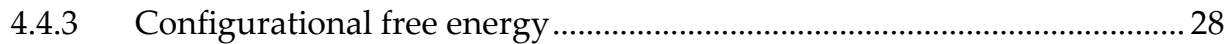




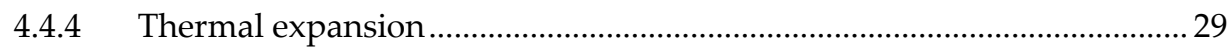

$5 \quad$ Elastic properties .............................................................................................................. 31

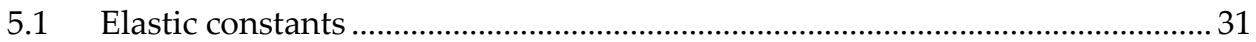

6 Magnetism: theory, methods, and materials............................................................... 35

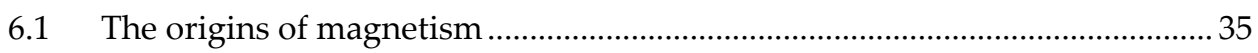

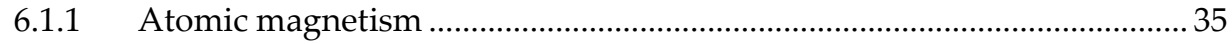

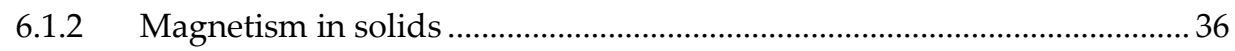

6.2 Magnetic energy and exchange interactions ................................................... 38

6.2.1 The Heisenberg Hamiltonian ……………………………………………..... 38

6.2.2 Magnetic exchange interactions ............................................................... 39

6.3 Magnetic MAX phases ...................................................................................... 40

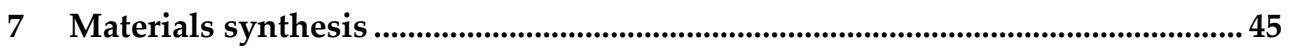

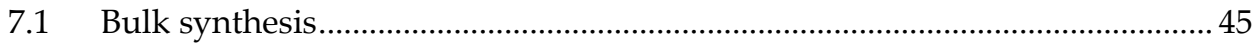

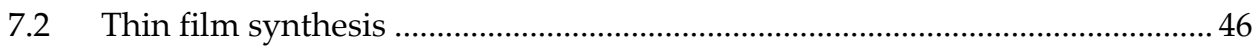

7.3 Differences between bulk and thin film synthesis.............................................. 48

8 Summary of included papers......................................................................................... 49

8.1 Paper I: Temperature dependent phase stability of nanolaminated ternaries

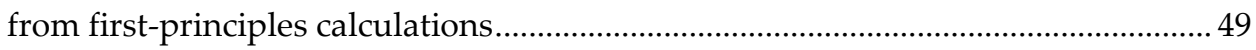

8.2 Paper II: Phase stability of the nanolaminates $\mathrm{V}_{2} \mathrm{Ga}_{2} \mathrm{C}$ and $\left(\mathrm{Mo1}-x \mathrm{~V}_{x}\right)_{2} \mathrm{Ga}_{2} \mathrm{C}$ from first-principles calculations ………………………………………………………..... 50

8.3 Paper III: First-principles calculations of the electronic, vibrational, and electronic properties of the magnetic laminate $\mathrm{Mn}_{2} \mathrm{GaC}$........................................... 51

8.4 Paper IV: Magnetic exchange interactions and critical temperature of the

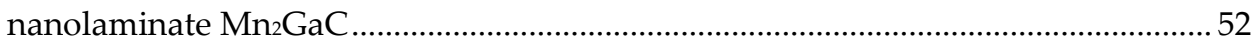

9 Contribution to the field..............................................................................................5 53

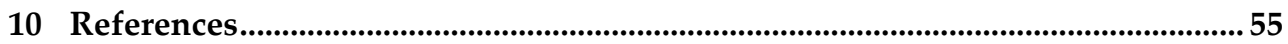




\section{Introduction}

\subsection{Computational materials science}

Throughout history, the search for and synthesis of new materials has mostly been a matter of experimental trial and error, with serendipity occasionally playing the leading role, as in the case of the discovery of, e.g., polytetrafluoroethylene (better known under the brand name Teflon). Since many existing technologies could benefit from materials with improved properties such as lighter weight, higher strength, and higher electrical conductivity, and since the creation of new technologies in some cases may even require these improvements, a faster, more systematic way of scanning through materials space is therefore highly desirable. Although experimental methods are being continuously refined, the greatest promise for a speedup of materials research comes from high-throughput computational screening, which has now become feasible thanks to the rapid advances in computer hardware as well as improvements in the efficiency and accuracy of software. This means that we have at our disposal a tool not only to help us screen for compounds, or phases, with interesting properties, but also to predict - unless they are already known to exist - whether these phases can be synthesized, or if they are likely to be outcompeted by the formation of some of the other phases within their respective materials systems. These predictions can then be used to guide experiment, potentially leading to drastic cuts in the time spent on synthesizing phases with few or no technologically useful properties, or on fruitless attempts to synthesize phases that calculations would have shown to be unstable with respect to competing phases.

While it seems like a certain bet that high-throughput computational screening will make up a significant part of materials research in the foreseeable future, this approach has, of course, yet to be perfected. One of the main problems is that the various software used to carry out the calculations are implementations of theoretical frameworks that are not exact. Also, calculating the behavior even of small atomic clusters is currently feasible only using modern supercomputers, as smaller computers still have ways to go before they are powerful enough to solve the underlying equations within acceptable timeframes.

In order to lessen the demand for computational resources, the calculations are often simplified by approximating the conditions to which the phases under investigation are subjected. One particularly common approximation is that the temperature at 
phase formation is at absolute zero. While this speeds up the calculations significantly, it does not reflect experimental conditions very well, since synthesis usually occurs well above room temperature. Nevertheless, for at least one class of materials the results of phase stability calculations under this approximation have so far proven remarkably accurate: the class of so-called MAX phases, which are nanolaminated materials with a hexagonal lattice occupied by $M, A$, and $X$ atoms (transition metals, $A$-group elements, and carbon and/or nitrogen, respectively). Paper I in this thesis is a first attempt at providing an explanation for this accuracy, using computational methods. The chosen materials system is the theoretically and experimentally wellexplored Ti-Al-C ternary system, and the phases for which stability is investigated are the three MAX phases $\mathrm{Ti}_{2} \mathrm{GaC}, \mathrm{Ti}_{3} \mathrm{GaC}_{2}$, and $\mathrm{Ti}_{4} \mathrm{GaC}_{3}$, where the first two are known to exist and for which $0 \mathrm{~K}$ calculations correctly indicate phase stability.

In paper II we explore the stability of two MAX-like phases, the nanolaminates $\mathrm{V}_{2} \mathrm{Ga}_{2} \mathrm{C}$ and $\left(\mathrm{Mo}_{1-x} \mathrm{~V}_{x}\right)_{2} \mathrm{Ga}_{2} \mathrm{C}$, the latter which is particularly interesting as potential parent material for synthesis of novel two-dimensional MXenes. For the former phase, we make use of the $0 \mathrm{~K}$ approximation. However, from previous work on MAX phases with solid solutions on the $M$ sublattice it is known that the configurational entropy gives a significant contribution to the Gibbs free energy at elevated temperatures. For $\left(\mathrm{Mo1}-\mathrm{x} \mathrm{V}_{x}\right)_{2} \mathrm{Ga}_{2} \mathrm{C}$, this particular temperature dependent effect is consequently included in the calculations.

In paper III and IV we use computational methods to characterize the recently discovered MAX phase $\mathrm{Mn}_{2} \mathrm{GaC}$, which is one of the first magnetic MAX phases to be synthesized. The phase is characterized with respect to its electronic, vibrational, and elastic properties. We also predict the magnetic critical order-disorder temperature.

\subsection{Outline}

This thesis is structured as follows. Chapter 2 gives an introduction to MAX phases in general, as well as to the particular MAX and MAX-like phases investigated in the four included papers. In chapter 3, density functional theory, which is the theoretical framework at the heart of all calculations carried out herein, is presented. Thermodynamic phase stability is the subject of chapter 4 , which discusses the conditions for stability, and the effects of temperature. Chapter 5 contains a short discussion of elastic properties, while chapter 6 discusses magnetism, with a particular focus on magnetic MAX phases. And although this thesis is based entirely on computer simulations, a chapter on materials synthesis, chapter 7 , is also included in 
order to give some idea of how the materials discussed have been - or may be realized experimentally. Chapter 8 contains summaries of the four included papers, and, finally, chapter 9 lists the contributions to the field of materials science that the results presented in these papers make. 


\section{MAX phases}

The $\mathrm{M}_{n+1} \mathrm{AX}_{n}(\mathrm{MAX})$ phases, where $n=1-3$, together constitute a class of materials that to date contains more than 70 phases. They are characterized by a hexagonal crystal lattice with a vertical lattice parameter $c$ typically 4-8 times longer than the basal plane lattice parameter $a$, and with individual atomic layers stacked on top of each other, as shown in Fig. 2.1 (a)-(c).

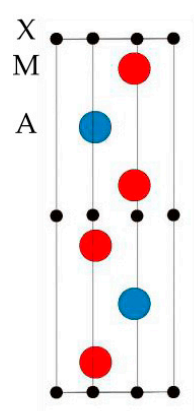

(a)

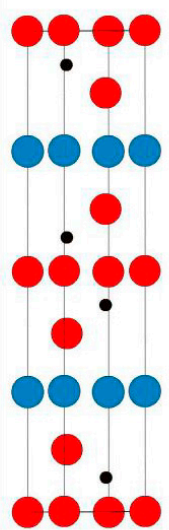

(b)

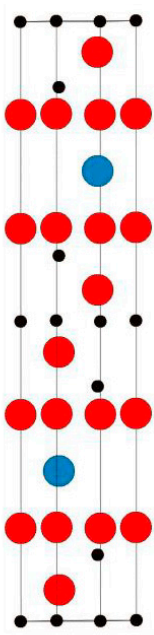

(c)

Figure 2.1 (a) The unit cell of an $\mathrm{M}_{2} \mathrm{AX}$ phase. (b) $\mathrm{M}_{3} \mathrm{AX}_{2}$. (c) $\mathrm{M}_{4} \mathrm{~A} X_{3}$.

All MAX phases are made up of $M, A$, and $X$ elements, which are found in the highlighted regions of the periodic table in Fig. 2.2. The $M$ elements consist of transition metals, whereas the $A$ elements are $A$-group elements ${ }^{1}$ (mostly from groups 13 and 14). The $X$ elements are either carbon or nitrogen, or both. However, although any given MAX phase must contain both $M, A$, as well as $X$ elements, it does not necessarily have to be a ternary phase; for instance, several quaternary MAX phases with a solid solution on either one of the three different lattice sites have been synthesized, e.g., $(\mathrm{Cr}, \mathrm{Mn})_{2} \mathrm{AlC}[1], \mathrm{Ti}_{3}(\mathrm{Sn}, \mathrm{Al}) \mathrm{C}_{2}$ [2], and $\mathrm{Ti}_{2} \mathrm{Al}(\mathrm{C}, \mathrm{N})$ [3].

\footnotetext{
${ }^{1}$ This naming convention is now discouraged in favor of just using the group numbers, but it is still widely used in the MAX phase literature, which is why it is also used here.
} 


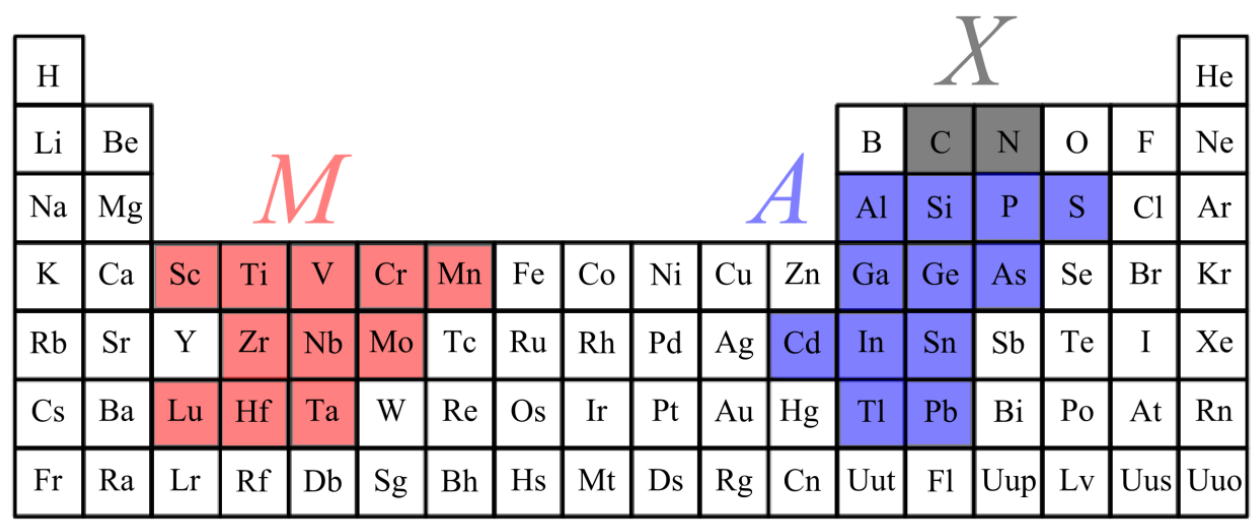

Figure 2.2 The $M, A$, and $X$ elements forming all currently known MAX phases.

By far the most common MAX phases are the ones with the formula $\mathrm{M}_{2} \mathrm{AX}(n=1)$, which can be described structurally as being made up of single $\mathrm{M}_{6} \mathrm{X}$ octahedra interleaved between the $A$ layers; around 50 ternary and a few quaternary $\mathrm{M}_{2} \mathrm{AX}$ phases have been synthesized to date. Significantly less common are the $\mathrm{M}_{3} \mathrm{AX}_{2}(n=2)$ and $\mathrm{M}_{4} \mathrm{AX}_{3}(n=3)$ phases, which are made up of two and three consecutive $M_{6} X$ octahedra between the $A$ layers, respectively. Reports of higher order MAX phases $(n \geq 4)$ are rare; in fact, there is a disagreement over whether these reports provide strong enough evidence to conclusively show that they actually exist [4].

Recently, the MAX-like phase $\mathrm{Mo}_{2} \mathrm{Ga}_{2} \mathrm{C}$ was synthesized [5]. This is a phase which is possibly the first member of an entire class of phases with the formula $M_{n+1} A_{n+1} X_{n}$. Its structure is very similar to that of a $\mathrm{M}_{2} \mathrm{AX}$ phase, the only difference being that it has an extra $A$ layer interleaved between the $\mathrm{M}_{6} \mathrm{X}$ octahedra, as seen in Fig. 2.4 [6].

Also related to the MAX phases is the class of so-called MXenes, which has garnered quite a lot of interest recently. MXenes are derived from MAX phases by etching of the A-layer, which leaves two-dimensional, nanometer thick MX sheets similar to graphene (hence the suffix "-ene") [7]. 


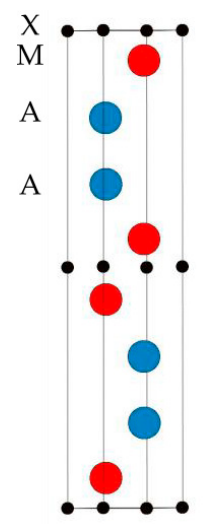

Figure 2.4 The unit cell of a MAX-like $\mathrm{M}_{2} \mathrm{~A}_{2} \mathrm{X}$ phase.

\subsection{History}

In the 1960s, Nowotny et al. synthesized and characterized the first MAX phases, initially called $H$-phases ${ }^{2}$. All phases had a 211 stoichiometry, and among them were $\operatorname{Ti}_{2} \mathrm{AX}(A=\mathrm{Al}, \mathrm{Ga}$, In, and $X=\mathrm{C}, \mathrm{N}), \mathrm{V}_{2} \mathrm{AC}(A=\mathrm{Al}, \mathrm{Ga}, \mathrm{Ge})$, and $\mathrm{Cr}_{2} \mathrm{AC}(A=\mathrm{Al}, \mathrm{Ga}, \mathrm{Ge})[8]$. Later Nowotny et al. also reported on the experimental synthesis of the two $\mathrm{M}_{3} \mathrm{AX}_{2}$ phases $\mathrm{Ti}_{3} \mathrm{SiC}_{2}$ and $\mathrm{Ti}_{3} \mathrm{GeC}_{2}[9,10]$. However, interest in further research on these phases was relatively low for almost 30 years following this work [11]. It was not until the 1990s, when Barsoum and El-Raghy synthesized and characterized highly phase pure $\mathrm{Ti}_{3} \mathrm{SiC}_{2}$ that MAX phases - it was at this time that the term "MAX phases" was coined - began to receive more attention [12]. From Barsoum and El-Raghy's work on $\mathrm{Ti}_{3} \mathrm{SiC}_{2}$ as well as on several other MAX phases including $\mathrm{Ti}_{4} \mathrm{AlN}_{3}$, it became clear that many of the phases within this class of materials possess quite remarkable physical properties, with considerable potential for technological applications - a realization that is now the main driver of MAX phase research.

\subsection{Properties and applications}

What makes MAX phases so promising is the fact that they exhibit a mix of metallic and ceramic properties. This mix can be attributed partly to the layered structure, and partly to the $M-A$ and $M-X$ bonds. The former bonds are predominantly metallic in

\footnotetext{
${ }^{2}$ It is sometimes claimed that $\mathrm{H}$ stands for "Hägg", the name of a class of interstitial compounds with close-packed metal sublattices. But as discussed by Eklund et al. in Ref [4], this is not the case; instead, " $\mathrm{H}$ " was likely just chosen so that it would fit into an alphabetical naming scheme already in use for other phases, such as " $\mathrm{D}$ ", " $\mathrm{E}$ ", and "G" phases.
} 
character and relatively weak, and the latter are predominantly covalent and relatively strong. The metallic aspects of the MAX phases are reflected in, e.g., an often high electrical and thermal conductivity, high fracture toughness, a resistance to thermal shock, and high machinability, i.e., they can easily be cut, drilled, polished etc. Common electrical and thermal conductivities of MAX phases are $\sim 1.4-5 \mu \Omega \cdot \mathrm{m}$ (at room temperature) and $12-60 \mathrm{~W} / \mathrm{K} \cdot \mathrm{m}$, respectively, which are numbers comparable to those for pure titanium [13]. However, unlike metals, but like ceramic materials, MAX phases are, in general, quite stiff and resistant to wear as well as to oxidation and creep. They also retain much of their strength even at high temperatures $\left(>1000{ }^{\circ} \mathrm{C}\right)$.

The excellent high temperature properties paired with the fracture toughness of some MAX phases (as opposed to ceramic materials, which are resistant to heat but brittle) means that they could possibly be used in the construction of internal combustion engines (Fig. (2.3 (a)) that can operate at higher temperatures than is currently possible, thus making them more efficient. Examples of other potential applications are as coatings of electrical contacts (which requires, e.g., heat and oxidation resistance, as well as good conductivity), rapidly spinning objects such as turbine blades (resistance to creep) (Fig. 2.3 (b)), and cutting tools (wear resistance) (Fig. 2.3 (c)). MAX phases have also been suggested for use in medical implants [14].

However, just as for other exciting materials such as, for instance, graphene, there are still challenges to be overcome with respect to industrial-scale production of MAX phases, whether it is in bulk or thin film form. Nevertheless, a few commercial MAX phase products already exist. Sandvik Heating Technology AB markets both $\mathrm{Ti}_{2} \mathrm{AlC}$ and $\mathrm{Ti}_{3} \mathrm{SiC}_{2}$ in powder form and in the form of solid targets for use in the production of thin films (Fig. 2.3 (d)); however, the coatings themselves are nanocomposites, and thus not exclusively composed of MAX phases. 
(a)

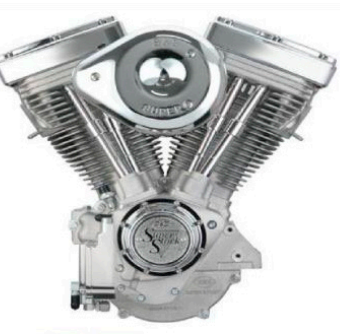

(c)

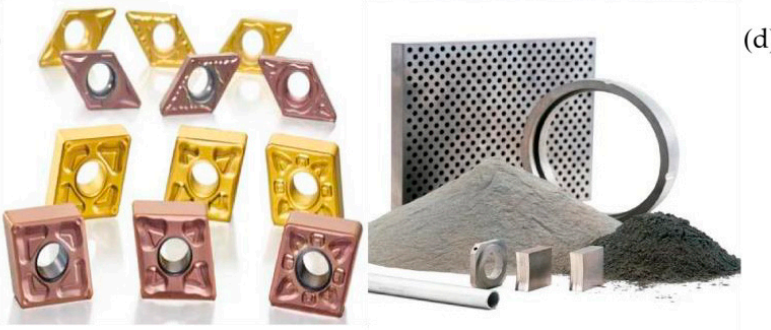

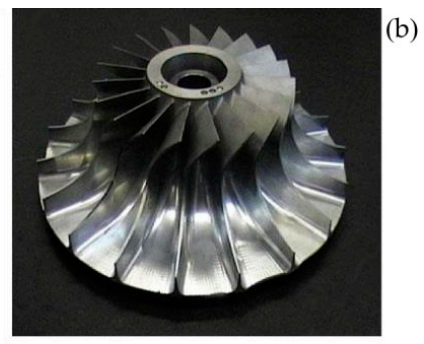

(d)

Figure 2.3 (a)-(c) Potential applications of MAX phases. Images taken from Refs. [15-17]. (d) Solid targets and powder of Maxthal 211 and $312\left(\mathrm{Ti}_{2} \mathrm{AlC}\right.$ and $\left.\mathrm{Ti}_{3} \mathrm{SiC}_{2}\right)$ manufactured by Sandvik Heating Technology AB.

\subsection{Materials investigated in this thesis}

Four MAX phases and two MAX-like phases are investigated in this thesis: $\operatorname{Ti}_{n+1} \mathrm{AlC}_{n}$ (for $n=1-3$ ) in paper $\mathrm{I}, \mathrm{V}_{2} \mathrm{Ga}_{2} \mathrm{C}$ and $\left(\mathrm{Mo}_{1-x} \mathrm{~V}_{x}\right)_{2} \mathrm{Ga}_{2} \mathrm{C}$ in paper II, and $\mathrm{Mn}_{2} \mathrm{GaC}$ in paper III and IV.

\subsection{1 $\mathrm{Ti}_{2} \mathrm{AlC}, \mathrm{Ti}_{3} \mathrm{AlC}_{2}$, and $\mathrm{Ti}_{4} \mathrm{AlC}_{3}$}

Due in large part to the availability of the three different elements, the Ti-Al-C ternary system is both theoretically and experimentally well-explored. On top of the single element phases, it contains several Ti-C and Ti-Al binaries, as well as one $\mathrm{Al}-\mathrm{C}$ binary $\left(\mathrm{Al}_{4} \mathrm{C}_{3}\right)$. Ternary phases are the inverse perovskite ${ }^{3} \mathrm{Ti}_{3} \mathrm{AlC}$ and the two MAX phases $\mathrm{Ti}_{2} \mathrm{AlC}$ and $\mathrm{Ti}_{3} \mathrm{AlC}_{2}$, all three for which phase stability calculations and experiment are in agreement.

As stated in Sec. 2.1, Ti2 AlC was among the first MAX phases to be synthesized. It was also one of the first MAX phases to be available for commercial use. The first reports on $\mathrm{Ti}_{3} \mathrm{AlC}_{2}$, on the other hand, did not show up until the 1990's. A very interesting property of both $\mathrm{Ti}_{2} \mathrm{AlC}$ and $\mathrm{Ti}_{3} \mathrm{AlC}_{2}$ from an applications perspective is that when they

${ }^{3}$ A class of cubic ternary compounds that shows lots of promise for photovoltaic applications. 
are heated, a layer of $\mathrm{Al}_{2} \mathrm{O}_{3}$ forms on the surface as $\mathrm{Al}$ reacts with - if present - $\mathrm{O}$ atoms. This layer strongly adheres to the underlying MAX phase and protects against further oxidation even after repeated thermal cycling, thus making $\mathrm{Ti}_{2} \mathrm{AlC}$ and $\mathrm{Ti}_{3} \mathrm{AlC}_{2}$ suitable as protective coatings of high-temperature applications in oxidizing environments. However, for reasons that include an increased probability of $\mathrm{Al}_{2} \mathrm{O}_{3}$ formation in $\mathrm{Ti}_{2} \mathrm{AlC}$ as compared to $\mathrm{Ti}_{3} \mathrm{AlC}_{2}$, explained by the higher concentration of $\mathrm{Al}$ in the former phase, $\mathrm{Ti} 2 \mathrm{AlC}$ would likely be preferred over $\mathrm{Ti}_{3} \mathrm{AlC}_{2}$ for such applications $[13,18,19]$.

While the theoretical results for $\mathrm{Ti}_{4} \mathrm{AlC}_{3}$ in paper I do not clearly indicate either stability or instability, the fact that no reports on the synthesis of this phase exist and that there is a lack of reports on synthesized $\mathrm{M}_{4} \mathrm{AX}{ }_{3}$ phases in general, renders likely the conclusion that it is indeed not possible to synthesize this phase.

\subsection{2 $\mathrm{V}_{2} \mathrm{Ga}_{2} \mathrm{C}$ and $\left(\mathrm{Mo} 1-x \mathrm{~V}_{x}\right)_{2} \mathrm{Ga}_{2} \mathrm{C}$}

The recent discovery of $\mathrm{Mo}_{2} \mathrm{Ga}_{2} \mathrm{C}$ by Lai et al. [5] prompted the work in paper II of this thesis, in which the stability of $\mathrm{V}_{2} \mathrm{Ga}_{2} \mathrm{C}$ and $\left(\mathrm{Mo}_{1-x} \mathrm{~V}_{x}\right)_{2} \mathrm{Ga}_{2} \mathrm{C}$ is predicted. In the paper, $\left(\mathrm{Mo1}-x \mathrm{~V}_{x}\right)_{2} \mathrm{Ga}_{2} \mathrm{C}$ is discussed as a potential parent material for synthesis of the MXene $\left(\mathrm{Mo1}-x \mathrm{~V}_{x}\right)_{2} \mathrm{C}$.

The quaternary Mo-V-Ga-C phase diagram is very large, with a total of over 70 phases. MAX phases that have been synthesized in this system are $\mathrm{Mo}_{2} \mathrm{GaC}$ and $\mathrm{V}_{2} \mathrm{GaC}[8,20$, 21].

\subsection{3 $\quad \mathrm{Mn}_{2} \mathrm{GaC}$}

In the $\mathrm{Mn}-\mathrm{Ga}-\mathrm{C}$ system it is possible to find quite a few $\mathrm{MnC}$ and $\mathrm{MnGa}$ binaries, but no gallium carbides. The only ternary phase that has so far been synthesized is the MAX phase $\mathrm{Mn}_{2} \mathrm{GaC}$, which, due to the nonzero magnetic moments of the Mn atoms, is one of few known magnetic MAX phases. Since this phase is such a recent contribution to the MAX phase family, the understanding of its physical properties is still limited. The theoretical investigations in paper III and IV are some of the first attempts at changing this, and they also provide references for future experimental work.

Two other ternary MAX phases in the system, $\mathrm{Mn}_{3} \mathrm{GaC}_{2}$ and $\mathrm{Mn}_{4} \mathrm{GaC}_{3}$, as well as the inverse Perovskite $\mathrm{Mn}_{3} \mathrm{GaC}$, have all been shown through first-principles calculations to be outcompeted by other phases, and it is therefore unlikely that any of these ternaries will be synthesized in the future. 


\section{Density functional theory}

The theoretical framework underlying all calculations in this thesis is density functional theory (DFT), which provides a practical, first-principles based approach to the simulation of large systems of interacting particles. The use of DFT has increased rather dramatically over the past two decades, and today it is the premier tool for theoretical materials science research.

\subsection{The energy of a system of interacting particles}

The starting point in DFT is the Hamiltonian for a system of interacting electrons and atomic nuclei, which can be written as

$$
\begin{gathered}
\widehat{H}=-\frac{\hbar^{2}}{2 m_{e}} \sum_{i} \nabla_{i}^{2}+\sum_{i} v_{e x t}\left(\mathbf{r}_{i}\right)+\frac{1}{2} \sum_{i \neq j} \frac{e^{2}}{\left|\mathbf{r}_{i}-\mathbf{r}_{j}\right|} \\
-\sum_{I} \frac{\hbar^{2}}{2 M_{I}} \nabla_{I}^{2}+\frac{1}{2} \sum_{I \neq J} \frac{Z_{I} Z_{J} e^{2}}{\left|\mathbf{R}_{I}-\mathbf{R}_{J}\right|}
\end{gathered}
$$

In this expression, the first term is the total kinetic energy of the electrons, while the second and third terms are the total potential energy due to the electron-ion and the electron-electron interactions, respectively. The last two terms are the total kinetic energy of the ions, and the total potential energy of the ion-ion interactions.

However, the problem of calculating the energy of the system is mainly a problem of calculating the total electronic energy, thanks to the so-called Born-Oppenheimer approximation. According to this approximation, the surrounding electrons, due to their much smaller masses, will reach their equilibrium states almost instantly upon small changes in the positions of the ions. This entails that the wave function describing the entire system can be written as the product of an electronic and an ionic part, and that the ionic part can be treated as a constant while calculating the electronic energy. The ionic part can be treated within a classical framework, whereas the electronic part requires quantum mechanical calculations due to the complicated dynamics of the electronic interactions. 
We may thus limit the discussion to a consideration of the Hamiltonian representing the total energy of a collection of electrons moving in the external electric potential $v_{\text {ext }}(\mathbf{r})$ generated by a set of static, positively charged ions:

$$
\widehat{H}=-\frac{\hbar^{2}}{2 m_{e}} \sum_{i} \nabla_{i}^{2}+\sum_{i} v_{e x t}\left(\mathbf{r}_{i}\right)+\frac{1}{2} \sum_{i \neq j} \frac{e^{2}}{\left|\mathbf{r}_{i}-\mathbf{r}_{j}\right|} .
$$

Since the ions are static, $v_{\text {ext }}(\mathbf{r})$ must be static as well, and the challenge of calculating the electron-ion interaction energy is therefore significantly reduced. The electronelectron interaction energy, on the other hand, poses a considerable computational challenge, since the motion of each electron is affected by the simultaneous motion of every other electron in the system.

DFT was developed in order to circumvent this so-called quantum mechanical manybody problem, which becomes a significant practical obstacle for systems larger than $N \sim 10$ particles. Even at such modest system sizes, the electronic many-body wave function $\Psi_{e}$ required to correctly describe the motion of the surrounding electrons is, with its multiple degrees of freedom, complex enough that solving the Schrödinger equation becomes prohibitively expensive in terms of computational resources.

In contrast, DFT relies in principle only on the electron density $n(\mathbf{r})$, which has three spatial degrees of freedom, and the required storage space and computational time thus scale much slower, the latter approximately as $N^{3}$. Currently this allows for calculations of systems comprised of up to about 1000 atoms; however, further development of DFT may improve this scaling, which would allow for computational treatment of even larger atomic systems.

\subsection{The Hohenberg-Kohn Theorems}

At the root of DFT are the two so-called Hohenberg-Kohn theorems, named after their originators, Pierre Hohenberg and Nobel laureate Walter Kohn [22]. The first H-K theorem states that the external potential $v_{\text {ext }}(\mathbf{r})$ of a system of interacting electrons is uniquely determined by its ground state density $n(\mathbf{r})$, up to an additive constant. In other words, for a given ground state electron density, there is only one possible external potential. Since $v_{\text {ext }}(\mathbf{r})$ in turn uniquely determines the Hamiltonian of the system and thus the many-body wave function, it follows from the first $\mathrm{H}-\mathrm{K}$ theorem that $n(\mathbf{r})$ therefore completely determines the system's ground state properties, 
including the ground state energy, which is the quantity of primary interest in this thesis.

From this follows the second Hohenberg-Kohn theorem, which states that for any given external potential $v_{\text {ext }}(\mathbf{r})$, one can define a functional - i.e., a function whose input argument is another function, and whose output is a number - of the density,

$$
E_{v}[n(\mathbf{r})]=F_{H K}[n(\mathbf{r})]+V\left[v_{\text {ext }}(\mathbf{r}), n(\mathbf{r})\right]
$$

where the Hohenberg-Kohn functional $F_{H K}[n(\mathbf{r})]$ accounts for the electronic kinetic energy and for all electron-electron interactions. The theorem further states that for a particular $v_{\text {ext }}(\mathbf{r})$, the functional defined in Eq. (3.3) is minimized by the ground state density $n(\mathbf{r})$ associated with this potential. This variational principle can be formulated mathematically as

$$
\begin{aligned}
E_{v}[n(\mathbf{r})]=F_{H K} & {[n(\mathbf{r})]+V\left[v_{\text {ext }}(\mathbf{r}), n(\mathbf{r})\right] } \\
< & F_{H K}\left[n^{\prime}(\mathbf{r})\right]+V\left[v_{\text {ext }}(\mathbf{r}), n^{\prime}(\mathbf{r})\right],
\end{aligned}
$$

where $n^{\prime}(\mathbf{r})$ is any density separate from $n(\mathbf{r})$.

However, while the two $\mathrm{H}-\mathrm{K}$ theorems prove that the electron density can in principle be used as the basic variable, they do not immediately lead to a practical recipe for calculating the ground state properties, since they do not give the exact form of the $\mathrm{H}$ K functional $F_{H K}[n(\mathbf{r})]$. To date, this form is still unknown. Even so, the realization that the energy of a system of interacting particles can be expressed as a functional of the density has proven very fruitful, as it has led to a reformulation of the intractable many-body problem into the significantly less demanding problem of calculating the energy of a system of non-interacting particles.

\subsection{The Kohn-Sham approach to DFT}

Today, DFT is more or less synonymous with the approach developed by Kohn and Sham [23]. This approach rests on the assumption that for any system of interacting particles, it is possible to construct an auxiliary, fictitious system of non-interacting particles whose energy is minimized by the same density as the ground state density of the real system. While there is no general proof for this assumption, the fact that it has so far held up remarkably well makes the Kohn-Sham approach an invaluable tool for materials scientists. 
The starting point of the Kohn-Sham approach is to write the H-K functional as the sum of the non-interacting part of the kinetic energy, $T_{S}[n(\mathbf{r})]$, the classical potential energy due to electron-electron Coulomb repulsion $J[n(\mathbf{r})]$ (also called the Hartree energy), and $E_{x c}[n(\mathbf{r})]$, the exchange-correlation energy, which contains the quantum mechanical parts of the kinetic and potential energy. Equation (3.3) thus becomes

$$
\begin{gathered}
E_{v}[n(\mathbf{r})]=T_{s}[n(\mathbf{r})]+J[n(\mathbf{r})]+E_{x c}[n(\mathbf{r})] \\
+V\left[v_{\text {ext }}(\mathbf{r}), n(\mathbf{r})\right] .
\end{gathered}
$$

The second step is to write an expression for the energy functional of the noninteracting system:

$$
E_{S}[n(\mathbf{r})]=T_{S}[n(\mathbf{r})]+V\left[v_{e f f}(\mathbf{r}), n(\mathbf{r})\right]
$$

In this system, the effective potential $v_{e f f}(\mathbf{r})$ serves as the external potential for the non-interacting particles.

Via Lagrange minimization of Eq. (3.5) and (3.6), it can be shown that the energy of both systems are minimized by the same density, if $v_{e f f}(\mathbf{r})$ is defined as the functional derivative with respect to $n(\mathbf{r})$ of the sum of $J[n(\mathbf{r})], E_{x c}[n(\mathbf{r})]$, and $V\left[v_{\text {ext }}(\mathbf{r}), n(\mathbf{r})\right]$ in Eq. (3.5), i.e., as

$$
v_{e f f}(\mathbf{r})=\frac{\delta J[n(\mathbf{r})]}{\delta n(\mathbf{r})}+\frac{\delta E_{x c}[n(\mathbf{r})]}{\delta n(\mathbf{r})}+v_{e x t}(\mathbf{r})
$$

where the first term is the Hartree potential $v_{H}(\mathbf{r})$, and the second term the exchangecorrelation potential $v_{x c}(\mathbf{r})$.

Just as for the interacting system, the exact form of the energy functional of the noninteracting system is unknown. However, even without this knowledge, calculating the energy of the latter system is much easier: instead of solving the many-body Schrödinger equation, one solves a set of $N$ Schrödinger-like so-called Kohn-Sham equations, given by

$$
\left(-\frac{\hbar^{2}}{2 m} \nabla^{2}+v_{e f f}(\mathbf{r})\right) \psi_{i}(\mathbf{r})=\epsilon_{i} \psi_{i}(\mathbf{r})
$$


where the $\psi_{i}$ 's are the Kohn-Sham orbitals, which can be expressed as, e.g., plane waves. The total energy $E_{s}[n(\mathbf{r})]$, which is the sum of the eigenenergies $\epsilon_{i}$, is minimized when the density used to construct $v_{e f f}(\mathbf{r})$ is reproduced by the sum of the squares of the K-S orbitals, i.e., when

$$
n(\mathbf{r})=\sum_{i}^{N}\left|\psi_{i}(\mathbf{r})\right|^{2}
$$

In other words, equation (3.8) has to be solved self-consistently with respect to the density, and the algorithm for this is described in Fig. 3.1.

1.

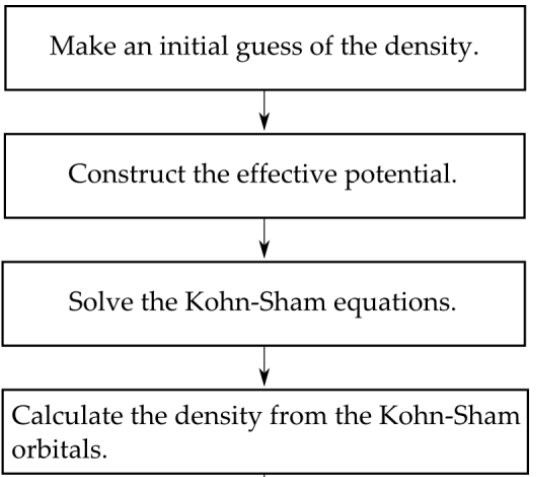
5. Compare new density to the initial density.
Are they equal?

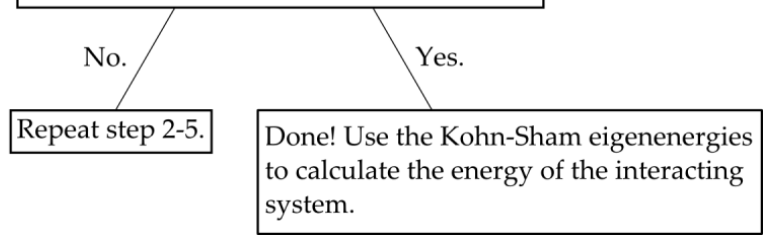

Figure 3.1 The Kohn-Sham self-consistent cycle.

The self-consistency requirement follows from the H-K theorems, which also hold true for the non-interacting system. Hence, just as for the interacting system, the Hamiltonian and therefore the Kohn-Sham orbitals of the non-interacting system are uniquely determined by the electron density. This means that, if the Kohn-Sham orbitals do indeed generate a density that matches the guessed initial density, then the energy of both systems is necessarily minimized. 
But even if the non-interacting and the interacting systems share a minimizing density, their ground state energies are not necessarily equal. However, a simple relation between the energy of the two systems exists. Together with the definition of the effective potential in Eq. (3.7), Eq. (3.6) can be rewritten as

$$
\begin{aligned}
T_{s}[n(\mathbf{r})] & =E_{s}[n(\mathbf{r})]-2 J[n(\mathbf{r})]-V\left[v_{x c}(\mathbf{r}), n(\mathbf{r})\right] \\
& -V\left[v_{\text {ext }}(\mathbf{r}), n(\mathbf{r})\right] .
\end{aligned}
$$

If the right-hand side of this equation is substituted into in Eq. (3.5), the expression for the energy of the interacting system becomes

$$
E_{v}[n(\mathbf{r})]=E_{s}[n(\mathbf{r})]-J[n(\mathbf{r})]+E_{x c}[n(\mathbf{r})]-V\left[v_{x c}(\mathbf{r}), n(\mathbf{r})\right]
$$

Finally, the total ground state energy of the particle system is given by adding $E_{v}[n(\mathbf{r})]$ to the potential energy of the atomic nuclei $E_{I I}$, which is just the classical ion-ion Coulomb repulsion:

$$
E_{t o t}=E_{v}[n(\mathbf{r})]+E_{I I}
$$

\subsection{Approximations of $E_{x c}$}

The main theoretical obstacle in DFT is the exchange-correlation energy, whose exact form is currently unknown. This entails that the energy of the non-interacting system, which depends on the functional derivative of $E_{x c}[n]$ according to Eq. (3.6) and (3.7), cannot be calculated exactly, a problem that carries over to the interacting system. However, the development of $E_{x c}[n]$ functionals is an active field of research, and the outcome so far has been several different and often useful approximations.

One of the simplest exchange-correlation functionals, the so-called local density approximation (LDA), takes advantage of the fact that exchange and correlation in many solids are local in nature, i.e., they are short range effects. This means that the exchange-correlation energy density functional (energy per particle) $\epsilon_{x c}[n]$, which integrates to $E_{x c}[n]$ according to the equation

$$
E_{x c}[n(\mathbf{r})]=\int \epsilon_{x c}[n(\mathbf{r})] n(\mathbf{r}) d r,
$$

can be reasonably approximated at each point $\mathbf{r}$ in space by the exchange-correlation 
energy density function $\epsilon_{x c}^{L D A}(n(\mathbf{r}))$ of a homogeneous electron gas (an electron gas with constant density). This is useful since approximate expressions for $\epsilon_{x c}^{L D A}(n(\mathbf{r}))$ is easier to derive than the still elusive $\epsilon_{x c}[n(\mathbf{r})]$.

$E_{x c}[n(\mathbf{r})]$, and hence $\epsilon_{x c}^{L D A}(n(\mathbf{r}))$, can be split into two separate parts with differing dependence on the density: one for exchange and one for correlation. For the exchange part there is a simple analytical expression, but the correlation part must generally be approximated, as exact expressions only exist in the limit of high density paired with weak correlation, and low density together with strong correlation. Since several different approximations for the correlation part exists, it is actually more correct to speak of local density approximations rather than a single LDA (although the latter is done here for convenience).

The LDA is exact for a homogenous electron gas and very accurate for spatially slowly varying densities [24], and compared to other $E_{x c}[n(\mathbf{r})]$ approximations it is also fairly computationally inexpensive. However, one of the main drawbacks of the LDA is that it tends to overestimate $E_{x c}[n(\mathbf{r})]$ for materials with strongly fluctuating densities, thus making the bonds between the atoms (and hence the lattice parameters in crystalline materials) too short. This may lead to erroneous predictions of a material's ground state structure and properties. An often cited example is Fe, where LDA calculations first yielded a nonmagnetic (or antiferromagnetic - the two magnetic configurations were degenerate in energy) face centered cubic (fcc) ground state structure, and in a later study an antiferromagnetic hexagonal close-packed (hcp) ground state structure, whereas it was known from experiment to be ferromagnetic and body centered cubic (bcc) [25, 26]. Also, the LDA cannot treat Van der Waals interactions, as they are inherently nonlocal with respect to electron correlation [27]. This makes the LDA unsuitable for calculations on materials like, e.g., graphite, where Van der Waals interactions make the graphene layers stick to each other. It should be noted, however, that in cases where the results from LDA calculations for individual phases are inaccurate only in a quantitative sense (e.g., the correct structure is found, but the lattice parameters are off by a significant amount), these results may still be used to accurately reflect trends in physical properties.

Building on the LDA is the gradient expansion approximation (GEA) and the generalized gradient approximation (GGA). As their names imply, both approximations include the gradient of the density, but they differ in that the former is merely a Taylor expansion of the LDA with respect to the density, whereas the latter is deliberately constructed to reproduce properties of the real exchange correlation 
energy functional such as the sum rule ${ }^{4}$, something which the GEA does not. Because of these differences, the GGA is more frequently used than the GEA - the latter functional does in fact perform worse than the LDA in many cases. The GGA, on the other hand, is considered a general improvement upon the LDA. While the GGA tends to underestimate $E_{x c}[n(\mathbf{r})]$ and make the atomic bonds too long, the magnitude of this error is usually smaller than the opposite error for the LDA. This and the fact that the GGA is comparable to the LDA with respect to the computational resources needed makes the GGA the better choice in most cases.

Just as for the LDA, various versions of the GGA exist; for MAX phase related firstprinciples calculations, the particular GGA developed by Perdew, Burke, and Ernzerhof (PBE, for short) [28] is probably the most popular one, and it is also the one used in this thesis. The PBE is a non-empirical (i.e., it does not use any parameter values derived from experiment) and thus transferable GGA that for MAX phases has proven to be relatively fast, and usually yields very accurate lattice parameters.

\subsection{Practical considerations}

In addition to the choice of exchange-correlation energy functional, the accuracy and speed of DFT calculations are affected by the size of the $\boldsymbol{k}$-point grid (with respect to the Kohn-Sham orbitals), and the plane wave energy cutoff.

\subsection{1 $k$-point convergence}

A suitable basis set in which to express the Kohn-Sham orbitals in Eq. (3.8) has to be decided upon before running through the K-S self-consistent cycle. A basis set is any set of linearly independent functions that can be combined to represent every possible state of a particle or system of particles. A common choice of such functions when working with periodic potentials, e.g., external potentials generated by the periodically arranged atomic nuclei in crystalline materials, is so-called Bloch waves. A Bloch wave consists of a plane wave part multiplied by a function which is of the same periodicity as the electron density (which in turn is of the same periodicity as the potential) $)^{5}$. It can be written as

$$
\psi_{j, \boldsymbol{k}}(\mathbf{r})=e^{i \boldsymbol{k} \cdot \mathbf{r}} u_{j, \boldsymbol{k}}(\mathbf{r})
$$

\footnotetext{
${ }^{4}$ This rule says that a spatial integration over the so-called exchange-correlation hole, which is a local decrease in the density around an electron with respect to the average density, should be equal to -1 . ${ }^{5}$ Note that a Bloch wave $\psi_{j, k}$ does not itself have the same period as the potential, but is instead quasiperiodic, fulfilling the criterion that $\psi_{j, k}(\mathbf{r}+\mathbf{R})=g\left(\mathbf{r}, \psi_{j, k}(\mathbf{r})\right)$.
} 
where $j$ is the band index denoting the particular band in the first Brillouin zone to which the orbital belongs, and where $\boldsymbol{k}$ is the wave vector, or $\boldsymbol{k}$-point, associated with this orbital.

Plugging the Bloch waves into Eq. (3.8) and (3.9) gives both the eigenenergies $\epsilon_{j}(\boldsymbol{k})$ of the Kohn-Sham orbitals and the electron density, the latter for which the expression becomes

$$
n(\mathbf{r})=\sum_{j} \int_{B Z}\left|\psi_{j, \boldsymbol{k}}(\mathbf{r})\right|^{2} d^{3} k
$$

where the integral is taken over the first Brillouin zone, and the sum is taken over all occupied bands. Since the number of possible $\boldsymbol{k}$-points is infinite, a finite sample of these points is chosen. This means that the integral in Eq. (3.15) is replaced by a discrete sum over $\boldsymbol{k}$, so that the density is instead calculated by interpolation between the terms in this sum. As shown in Fig. 3.2, a large enough sample of $\boldsymbol{k}$-points has to be chosen in order to converge the ground state energy; a commonly used convergence criterion with respect to the $\boldsymbol{k}$-point grid is that the calculated energy from the two largest grids should differ by no more than $0.1 \mathrm{meV} /$ atom. How to choose this sample depends on the crystal structure and the length ratios between the lattice parameters. For a $M_{2} A X$ phase unit cell, where the ratio between the basal plane lattice vectors $\boldsymbol{a}_{1,2}$ and the vertical lattice vector $\boldsymbol{a}_{3}$ is $\sim 1 / 4$, a grid with four times as many $\boldsymbol{k}$-points along the $\boldsymbol{k}$ space basal plane axes should be used as compared to the vertical axis, since the respective lengths of the basis vectors spanning $\boldsymbol{k}$-space,

$$
\begin{gathered}
\boldsymbol{b}_{1}=2 \pi \frac{\boldsymbol{a}_{2} \times \boldsymbol{a}_{3}}{\boldsymbol{a}_{1} \cdot\left(\boldsymbol{a}_{2} \times \boldsymbol{a}_{3}\right)} \quad \boldsymbol{b}_{2}=2 \pi \frac{\boldsymbol{a}_{3} \times \boldsymbol{a}_{1}}{\boldsymbol{a}_{1} \cdot\left(\boldsymbol{a}_{2} \times \boldsymbol{a}_{3}\right)} \\
\boldsymbol{b}_{3}=2 \pi \frac{\boldsymbol{a}_{1} \times \boldsymbol{a}_{2}}{\boldsymbol{a}_{1} \cdot\left(\boldsymbol{a}_{2} \times \boldsymbol{a}_{3}\right)}
\end{gathered}
$$

are related through $\left|\boldsymbol{b}_{1}\right|=\left|\boldsymbol{b}_{2}\right|$ and $\left|\boldsymbol{b}_{3}\right|=\frac{1}{4}\left|\boldsymbol{b}_{1,2}\right|$ when $\left|\boldsymbol{a}_{1}\right|=\left|\boldsymbol{a}_{2}\right|$. This yields the same $\boldsymbol{k}$-point density along all three axes. 


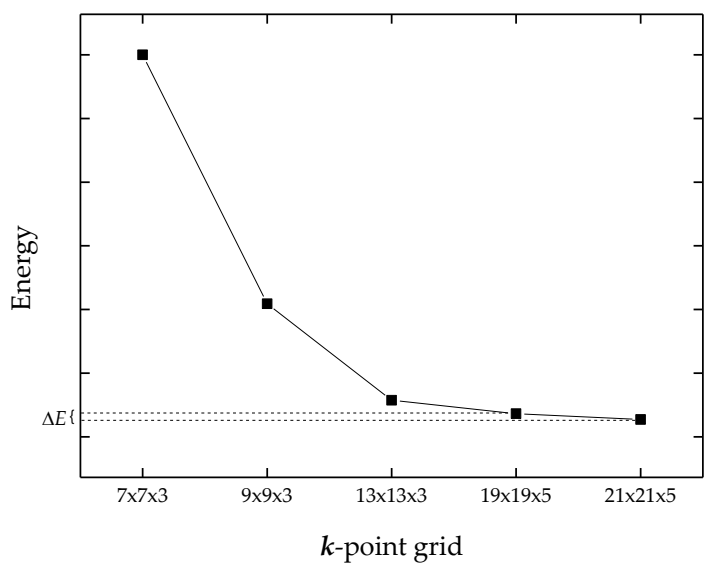

Figure 3.2. $k$-point convergence. For $\mathrm{MAX}$ phases convergence is reached when $\Delta E \leq 0.1 \mathrm{meV}$.

Using only a small sample of $\boldsymbol{k}$-points is possible for many materials since the magnitude of the Bloch waves is usually a slowly varying quantity; for metals, however, there are discontinuities in the integrand around the Fermi energy, which requires a larger $\boldsymbol{k}$-point sample [29]. Another factor speeding up the calculations is that if the $\boldsymbol{k}$-space associated with a particular crystal structure possesses several symmetries, as it is then enough to confine the calculations to only a part of the $\boldsymbol{k}$-point grid.

\subsubsection{Energy cutoff convergence and pseudopotentials}

Since the function $u_{k}(\mathbf{r})$ in Eq. (3.14) is periodic, it can be expanded in a Fourier series, thus yielding the expression

$$
u_{k}(\mathbf{r})=\sum C_{G} e^{i k \cdot r}
$$

Here, $\boldsymbol{G}$ is a reciprocal lattice vector given by

$$
\boldsymbol{G}=\lambda_{1} \boldsymbol{b}_{1}+\lambda_{2} \boldsymbol{b}_{2}+\lambda_{3} \boldsymbol{b}_{3}
$$

where $\lambda_{1,2,3}$ are positive integers. The Fourier coefficients $C_{\boldsymbol{G}}$ in Eq. (3.17) become smaller and less important as $|\boldsymbol{G}|$ becomes larger, and it is therefore possible to exclude large values of $|\boldsymbol{G}|$ in order to further speed up the calculations. The energy defined by 
the largest reciprocal lattice vector included in the calculations, $\left|\boldsymbol{G}_{\max }\right|$, is the so-called cutoff energy,

$$
\epsilon_{c u t}=\frac{\hbar^{2}}{2 m}\left|\boldsymbol{G}_{\max }\right|^{2}
$$

Thus, just as for the $\boldsymbol{k}$-point grid, the ground state energy must be converged with respect to $\epsilon_{c u t}$. The exact size of $\epsilon_{c u t}$ needed to reach convergence depends on how the potential in the core regions, i.e., the regions near the atomic nuclei, is treated. Since chemical bonds between atoms, whose nature and strength determine, e.g., the electric and mechanical properties of a phase, involve mainly the valence electrons, changes in the chemical environment do not affect the core electrons to any significant degree. This means that the difference in the ground state energy between two different phases is primarily given by the difference in energy between their respective valence states. Thus, the potential that the valence electrons feel from the core electrons can be regarded as fixed, and can therefore be combined with the external potential generated by the nuclei to form an effective ionic potential - a pseudopotential - that is much weaker than the real (external) potential in the core regions, but identical to it outside some cutoff radius $r_{c}$. The partially reduced strength of this potential makes it possible to replace the real wave functions of the valence electrons with pseudo wave functions that are smooth in the core regions instead of rapidly oscillating ${ }^{6}$, thus requiring fewer Fourier components than the real wave functions. Consequently, a smaller $\epsilon_{c u t}$ is possible.

Currently one of the most widely used methods for treating the wave functions in DFT in a computationally efficient manner is the projector augmented wave method (PAW), developed by Blöchl [30]. In PAW, which to a large extent is based on ideas from pseudopotential methods, the Kohn-Sham single particle (or all-electron) wave functions are decomposed into pseudo wave functions, which are smooth everywhere in space, and a sum consisting of rapidly oscillating wave functions that only contribute significantly in the core regions. PAW is the method of choice in all DFT calculations in this work.

\footnotetext{
${ }^{6}$ The oscillations are a consequence of the fact that the valence wave functions have to be orthogonal to the core wave functions, which are rapidly oscillating as well.
} 


\section{Phase stability calculations from first principles}

\subsection{Thermodynamic stability and metastability}

A central concept when discussing phase stability is the thermodynamic potential. In analogy with an electric potential, which is the energy required to bring a point charge from some reference point $A$ to point $B$, the thermodynamic potential is a measure of the energy it takes to form a phase under constant temperature $T$ and pressure $p$ from a reference state which can be defined by, e.g., the free constituent atoms at $T$ and $p$. This potential can be expressed as

$$
G(p, T)=E_{0}(V)+F_{e l}(T, V)+F_{v i b}(T, V)+F_{c}(T, V)+p V .
$$

This is the so-called Gibbs free energy, where the first term is the zero-temperature energy, the second and third terms are the electronic and vibrational contributions, respectively, which account for thermal excitations of electrons and phonons, and where the fourth term is the free configurational energy, which is nonzero only for configurationally disordered phases. The last term is the mechanical work the particle system has to perform against its surroundings to reach its final volume $V$.

Phase stability can be driven either by thermodynamics or by reaction kinetics. Thermodynamically driven phase stability is determined by calculating the Gibbs free energy of formation $\Delta G$, defined as the difference between the Gibbs free energy of the investigated phase and the Gibbs free energy of any polymorph ${ }^{7}$ or set of other competing phases with chemical compositions that, when properly weighted, combine to the same composition as that of the investigated phase (for example, for an $M_{2} A X$ phase, a set of competing phases might consist of the binaries MA and MX). The phase is thermodynamically stable if $\Delta G<0$ with respect to all possible competing phases and combinations thereof, i.e., if the Gibbs free energy of the investigated phase is at the global minimum of the Gibbs free energy landscape, as illustrated in Fig. 4.1. If this is indeed the case, the phase will tend to form spontaneously. In other words, just as there is a natural tendency for a negatively charged particle to minimize its potential energy by moving towards the positive source charge of an electrostatic field, there is

\footnotetext{
${ }^{7}$ That is, a phase with the same chemical composition as another phase, but with a different crystal structure.
} 
a natural tendency for a collection of atoms to combine into the phase, or set of phases, with the lowest value of $G$.

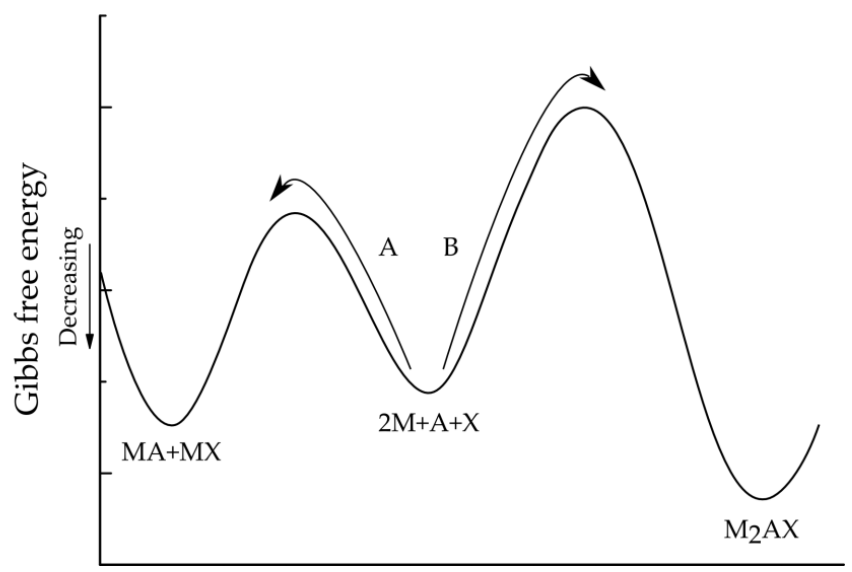

Figure 4.1. Hypothetical Gibbs free energy landscape for an M-A-X system. The $M_{2} A X$ phase is at the global minimum, while the respective sets of competing phases $\mathrm{MA}+\mathrm{MX}$ and $\mathrm{M}+\mathrm{A}+\mathrm{X}$ are found in local minima, i.e., they are metastable.

However, even if formation of the investigated phase is favored thermodynamically, it is still possible to end up with competing phases as very long-lived intermediate products - practically they may thus be seen as alternative end products. An important factor when it comes to phase stability is the activation energy, which is the energy needed to weaken or break the bonds between the constituent atoms of the initial phases in order to initiate the phase transition(s). In Fig. 4.1, there are two possible transition pathways with different activation energies (given by the height of the "bumps") and different end products. Although pathway A leads to an end product that is only metastable, i.e., at a local minimum of the Gibbs free energy landscape, it may still be favored over pathway B that leads to the global minimum of $G$, if the activation energy of pathway $\mathrm{A}$ is lower. Metastability is kinetically driven, which means that it depends on the rate of formation of the end products, a rate determined by the activation barrier together with external factors such as pressure and temperature. If this rate is higher for a metastable end product than for a competing, thermodynamically stable one, the former will be favored initially; however, the latter will form over time. The carbon allotrope diamond is an example of a metastable phase that, under the right ambient conditions, will transform into the thermodynamically 
stable allotrope graphite. In this thesis, however, the focus is on thermodynamically driven phase stability.

\subsection{Finding competing phases}

The first step in an investigation of the stability of a particular phase is to identify all competing phases, a task that requires careful study of the experimentally derived phase diagram of the relevant materials system. If the system is not very well-explored, additional hypothetical phases aside from the phase under investigation may have to be included. One way to decide which hypothetical phases to include is by looking at neighboring systems; if there are phases in these systems with crystal structures that cannot be found in the system of interest, it might be reasonable, as a first guess, to use these structures in the construction of hypothetical phases. However, if the neighboring systems are also not very well-explored, another approach for determining which hypothetical phases to include is to use evolutionary algorithms [31], although this has not been necessary in this work.

Previous phase stability studies focusing on MAX phases have often relied on incomplete sets of competing phases, leading to results that have not necessarily reflected the experimental data [32-34]. However, following Dahlqvist, Alling, and Rosen's recently developed linear optimization procedure to quickly determine the set of most competing phases, this has the potential to change [35]. This procedure is described and used in paper I to identify the set of most competing phases with respect to three $\mathrm{Ti}_{n+1} \mathrm{AlC}_{n}$ phases. It is also used in paper II.

\subsection{Thermodynamical phase stability at $T=0 \mathrm{~K}$}

Most first-principles based phase stability calculations are carried out using the approximations that the pressure is $0 \mathrm{GPa}$ and that the temperature is $0 \mathrm{~K}$, which reduces the Gibbs free energy given by Eq. (4.1) to the first term only, i.e., to the zerotemperature energy $E_{0}$. While these approximations describe a system quite different from real-world experimental conditions, the predictions of MAX phase stability have so far proven to be accurate, which is fortunate since the use of these approximations significantly cuts down the amount of required computational resources; for calculations on large supercells, for instance, they can lead to a decrease in computational time of several days. 


\subsubsection{Calculating $\boldsymbol{E}_{0}$}

Since the $0 \mathrm{~K}$ energy $E_{0}$ depends on the phase volume, identification of the equilibrium volume, for which the calculations yield the global minimum value of $E_{0}$, is needed. The equilibrium volume $V_{e q}$ is found when $E_{0}$ increases as one moves away from $V_{e q}$ in both directions, as seen in Fig. 4.2, and convergence has been reached with respect to the $\boldsymbol{k}$-point grid and the plane wave cutoff energy.

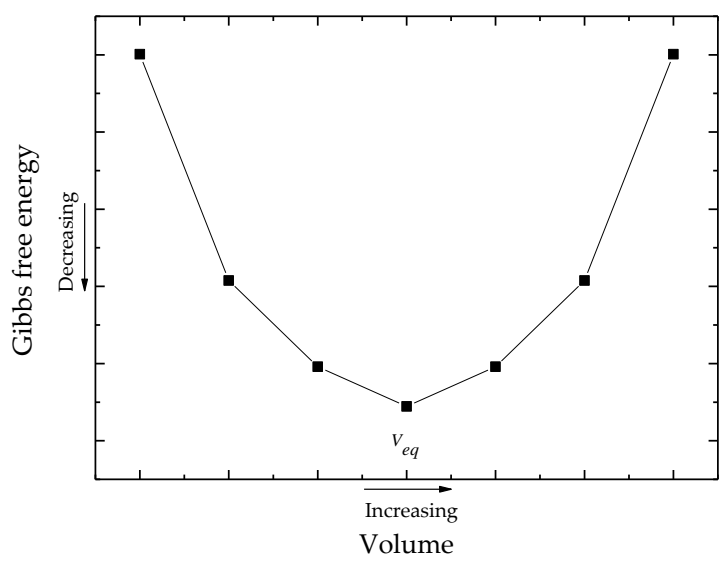

Figure 4.2. Gibbs free energy as a function of volume at $0 \mathrm{~K}$. The equilibrium volume $V_{e q}$ is found at the global minimum of the Gibbs free energy.

\subsection{Thermodynamical phase stability at $T>0 \mathrm{~K}$}

The accurate results from the $0 \mathrm{~K}$ calculations notwithstanding, until now no attempts at providing an explanation for this accuracy have been made. Such an explanation, which should reduce the uncertainty with respect to the reliability of future predictions of MAX phase stability, is provided in paper I.

When considering to which degree temperature dependent effects influence phase stability predictions, there are at least two more contributions to the Gibbs free energy in addition to $E_{0}$ that should be included in the calculations, namely the free electronic and free vibrational energy, i.e., the second and third terms in Eq. (4.1). In case of a disordered phase, the fourth term, the free configurational energy, also contributes, and should then be included as well. 


\subsubsection{Electronic free energy}

If the temperature is raised above $0 \mathrm{~K}$, some of the electrons are excited into states with higher energy. While these excitations are associated with a positive contribution $E_{e l}$ to the Gibbs free energy, it is counteracted by the simultaneous increase in the number of available electronic states and hence the electronic configurational entropy $S_{e l}$. In other words, the electronic contribution to the Gibbs free energy is given by the difference

$$
F_{e l}(V, T)=E_{e l}(V, T)-T S_{e l}(V, T) .
$$

The entropy term tends to dominate the expression even at very low temperatures, thus leading to a lowering in the Gibbs free energy; this is seen for the investigated phases in paper I.

\subsubsection{Vibrational free energy}

Atoms in a periodic lattice vibrate in concert about their equilibrium lattice positions, interacting with each other through electron-mediated forces. The complexity of these interactions makes the problem of directly calculating the total, temperature dependent vibrational free energy very difficult. However, similar to Kohn-Sham DFT, this problem can be mapped onto the equivalent but simpler problem of calculating the energy of a system of non-interacting quasiparticles called phonons - quantized modes of harmonic collective oscillations thusly named because of their mix of waveand particle-like behavior (just like particles, they carry momentum), in analogy with photons [36]. Phonons contribute significantly to, e.g., the heat capacity of a solid, as well as to thermal conduction, and just as for thermally excited electrons, they give a nonzero contribution to the Gibbs free energy consisting of an energy term and an entropy term:

$$
F_{v i b}(V, T)=E_{v i b}(V, T)-T S_{v i b}(V, T)
$$

Again there is a tendency for the entropy term to dominate the expression even at low temperatures, also seen paper I.

Equation (4.9) can also be written as 


$$
\begin{aligned}
F_{v i b}(V, T)= & \frac{1}{2} \sum_{\boldsymbol{q}, v} \hbar \omega_{\boldsymbol{q}, v} \\
& +k_{B} T \sum_{\boldsymbol{q}, v} \ln \left[1-\exp \left(-\hbar \omega_{\boldsymbol{q}, v} / k_{B} T\right)\right]
\end{aligned}
$$

where $\boldsymbol{q}$ is the phonon wave vector, and $v$ is the band index; the first term on the righthand side is the zero point energy, which, even though it is actually present at $0 \mathrm{~K}$, is typically neglected in $0 \mathrm{~K}$ calculations; it is often quite small, however. The allowed phonon frequencies $\omega_{\boldsymbol{q}, \boldsymbol{v}}$ can be calculated using, e.g., the linear response method, also known as density functional perturbation theory (DFPT) [37, 38].

In this thesis, DFPT as implemented in the VASP (Vienna ab initio simulation package) code is the method of choice. DFPT uses the fact that the first derivative of the electron density with respect to a shift in the positions of the ions - i.e., a perturbation of the external potential - is directly related to the second derivative of the energy with respect to this shift, which yields the interatomic force constants (IFCs) that are then plugged into the dynamical matrices to obtain the phonon frequencies. The derivative of the density for a given perturbation can be found through a self-consistent calculation analogous to the Kohn-Sham cycle in DFT, with the first derivatives of the unperturbed (ground state) K-S orbitals as solutions to the resulting eigenequations. In order to determine the phonon dispersion, self-consistent calculations have to be performed for several different phonon perturbations, each with a specific wave vector $\boldsymbol{q}$. In regular DFPT, the calculations are confined to the unit cell; however, VASP DFPT only calculates the frequencies at the $\Gamma$ point $(\boldsymbol{q}=0)$, which means that the vibrational free energy has to be converged with respect to supercell size. For the Ti-Al-C MAX phases in paper I, sufficient convergence was reached for $3 \times 3 \times 1$ supercells.

\subsubsection{Configurational free energy}

The configurational free energy comes into play for phases where one or more of the crystal sublattices are disordered because of, e.g., alloying or vacancies. Again there is an energetic cost associated with the excited, disordered state, and a counteracting term due to the increased entropy:

$$
F_{c}(V, T)=E_{c}(V, T)-T S_{c}(V, T) .
$$

Generating a disordered phase can be done - and has been done in this thesis - using the special quasirandom structure (SQS) method developed by Zunger et al. [39]. 


\subsubsection{Thermal expansion}

As indicated in Eq. (4.1), the Gibbs free energy depends on the volume of the phase. In the so-called harmonic approximation (HA) this dependence is neglected, which has the advantage that a significant amount of computational time is saved. However, as most materials expand with increasing temperature, the accuracy of the results may increase if the quasiharmonic approximation (QHA) is instead applied [40, 41].

In the QHA, the expansion is modeled in the following way: at a given temperature $T$, the volume of the phase (and hence the lattice parameters) is increased in a stepwise fashion, and at each volume the zero-temperature energy, the phonon dispersion, and the electronic contribution is calculated. These contributions are then added together, and an fit between the resulting data points yields the minimum of the Gibbs free energy and the equilibrium volume at $T$. When this process is repeated for several different temperatures, the result is usually in line with that shown in Fig. 4.2: the equilibrium volume increases with temperature, while the Gibbs free energy decreases.

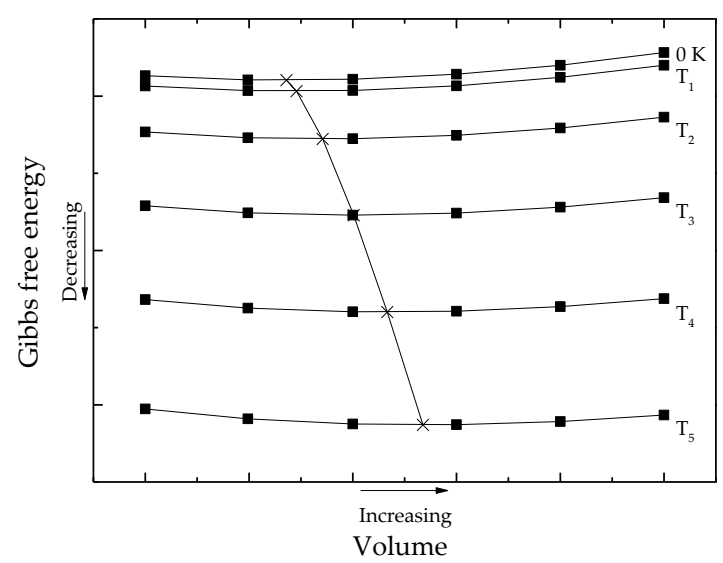

Figure 4.2. Gibbs free energy vs. volume with increasing temperature; each of the six curves represent the results at a given temperature, which increases strictly from $0 \mathrm{~K}$ to $T_{5}$. The line crossing the curves indicates the minimum energy and equilibrium volume at each temperature. 


\section{Elastic properties}

The elastic properties of a material, i.e., its tendency to return to its original shape after being subjected to tensile, compressive or shear stress, determine macroscopic properties such as friction, machinability, and ductility. They are of utmost importance for the structural integrity of components of a vast number of ubiquitous technologies such as cars, boats, airplanes, and buildings.

First-principles calculations of elastic properties have been carried out for most existing and several hypothetical MAX phases [42]. The results do not yet match experimental data as well as, e.g., calculations of the lattice parameters, which is likely mainly explained by the many approximations in DFT, but may also depend to some degree on the difficulties in experimentally achieving completely phase pure MAX phases [43]. The match between theoretical and experimental results, however, is generally good enough to yield useful information about trends in the elastic properties, as demonstrated in paper III in this thesis.

\subsection{Elastic constants}

The elastic properties of a crystalline phase can be obtained from its elastic constants, which are analogous to the spring constant $k$ in the one-dimensional formulation of Hooke's law, as they relate the applied stress, and hence the potential energy, to the strain induced in the lattice. However, since a crystal lattice can extend in all three spatial dimensions, several different elastic constants may be required for a full description of its elastic response.

The three-dimensional form of Hooke's law can be expressed in terms of a tensor equation, where the tensor elements are the different elastic constants $C_{i j}$. For a hexagonal phase such as a MAX phase, the independent elastic constants are $C_{11}, C_{12}$, $C_{13}, C_{33}$, and $C_{44}{ }^{8}$, which can be obtained by solving this equation. This is the so-called stress-strain method.

However, in this thesis the stress-energy method is used for obtaining the elastic constants. The starting point of this method is the application of five different strains

\footnotetext{
${ }^{8}$ In the literature this constant is sometimes labeled as $C_{55}$.
} 
to the crystal structure, each of which can be represented by a particular distortion matrix

$$
\left(\begin{array}{ccc}
1+\alpha_{1} & \alpha_{6} & \alpha_{5} \\
\alpha_{6} & 1+\alpha_{2} & \alpha_{4} \\
\alpha_{5} & \alpha_{4} & 1+\alpha_{3}
\end{array}\right)
$$

where the off-diagonal strain parameters $\alpha_{i}$ are pairwise identical due to the symmetry of the structure [44]. The basis of the strained structure, given by the product of the matrix (5.1) and the $3 \times 3$ basis vector matrix of the unstrained structure, then serves as input for a DFT calculation of its total energy $E(V, \alpha)$. This energy is given by the equation

$$
E(V, \alpha)=E\left(V_{0}, 0\right)+V_{0}\left(\sum_{i} \tau_{i} \alpha_{i} \xi_{i}+\frac{1}{2} \sum_{i, j} C_{i j} \alpha_{i} \xi_{i} \alpha_{j} \xi_{j}\right)
$$

where $E\left(V_{0}, 0\right)$ is the energy of the unstrained structure at the equilibrium volume $V_{0}$, $\tau_{i}$ are stress tensor elements, and $\xi_{i}$ are coefficients that equal 1 for $\alpha_{1,2,3}$ and, because of the pairwise symmetry, 2 otherwise. When all five strains have been applied, the result is five different equations derived from Eq. (5.2):

$$
\begin{gathered}
E(V, \alpha)-E\left(V_{0}, 0\right)=V_{0}\left(\left(\tau_{1}+\tau_{2}\right) \alpha+\left(C_{11}+C_{12}\right) \alpha^{2}\right), \\
E(V, \alpha)-E\left(V_{0}, 0\right)=V_{0}\left(\left(\tau_{1}-\tau_{2}\right) \alpha+\left(C_{11}-C_{12}\right) \alpha^{2}\right), \\
E(V, \alpha)-E\left(V_{0}, 0\right)=V_{0}\left(\tau_{3} \alpha+\frac{C_{33}}{2} \alpha^{2}\right), \\
E(V, \alpha)-E\left(V_{0}, 0\right)=V_{0}\left(\tau_{4} \alpha+2 C_{44} \alpha^{2}\right),
\end{gathered}
$$

and

$$
\begin{gathered}
E(V, \alpha)-E\left(V_{0}, 0\right) \\
=V_{0}\left(\left(\tau_{1}+\tau_{2}+\tau_{3}\right) \alpha\right. \\
\left.+\left(C_{11}+C_{12}+2 C_{13}+\frac{C_{33}}{2}\right) \alpha^{2}\right) .
\end{gathered}
$$


Since the right hand sides of Eqs. (5.3)-(5.7) are second degree polynomials with respect to $\alpha$, a way to extract the factors containing the elastic constants is to plot $E(V, \alpha)-E\left(V_{0}, 0\right)$ as a function of $\alpha$, as in Fig. (5.1), perform a quadratic fit on the data, and then take the second derivatives of the fitted curves. If the curves are not symmetric around $\alpha=0$, the chosen strains are either too large, or the structure is mechanically unstable; the latter case would indicate that the particular crystal structure is not the ground state structure.

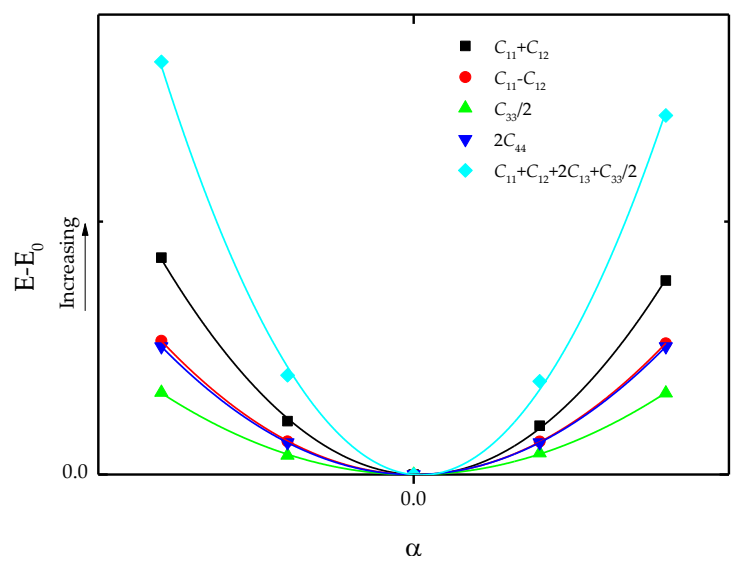

Figure 5.1. Energy vs. strain parameter for the five different strains yielding the five elastic constants for a MAX phase.

Once the elastic constants have been obtained, it is straightforward to derive, e.g., the bulk and shear modulus of the phase, since the moduli depend solely on the elastic constants, as shown in papers II and III. However, it is important to note that, if the investigated phase is magnetic, a careful search for the magnetic ground state configuration should be performed before calculating the elastic constants, since the magnetic configuration may influence the values of the elastic properties. This is seen, for instance, both in Ref. [45], where the bulk modulus of $\mathrm{Cr}_{2} \mathrm{AlC}$ is found to differ by about $10 \%$, depending on whether the phase is treated as nonmagnetic or antiferromagnetic, and in paper III, where the elastic properties of $\mathrm{Mn}_{2} \mathrm{GaC}$ are calculated. 


\section{Magnetism: theory, methods, and materials}

Magnetic materials can be used for a wide variety of purposes, from simple ones such as pinning a piece of paper to a refrigerator door, to more advanced ones such as navigation, electric power generation, magnetic cooling, and data storage.

\subsection{The origins of magnetism}

\subsubsection{Atomic magnetism}

An electron orbiting an atomic nucleus possesses two types of quantum mechanical angular momenta: orbital angular momentum, and spin. While the former is analogous to angular momentum in classical mechanics, spin is an intrinsic angular momentum with no classical analog; it does not depend on any form of classical rotational motion of the electron. The spin can take on only two values: $\hbar / 2$ or $-\hbar / 2$, often called "spin up" and "spin down".

Both types of angular momenta are associated with a magnetic dipole moment, and if the atom contains one or more unpaired electrons, i.e., electrons for which the sets of quantum numbers $N$ (principal), $l$ (azimuthal), and $m_{l}$ (magnetic) are unique, the electron magnetic moments sum up to a net atomic magnetic moment. In this case, the atom is said to be paramagnetic. If a paramagnetic atom is subjected to an external magnetic field, it experiences an attractive force which tends to align the atomic moment with this field.

Atoms with no unpaired electrons and thus no net magnetic moment, on the other hand, are purely diamagnetic. While diamagnetic atoms are nonmagnetic in the absence of an external magnetic field, a magnetic moment is induced if a magnetic field is applied. The polarity of the induced field is opposite to that of the applied field, which leads to the atom experiencing a repulsive force.

Except for hydrogen ${ }^{9}$, all atoms, even paramagnetic ones, are actually diamagnetic to some degree due to the presence of paired core electrons; however, in paramagnetic atoms the repulsive diamagnetic response to an externally applied magnetic field is masked by the much stronger attractive paramagnetic response.

\footnotetext{
${ }^{9} \mathrm{H}$ atoms combine into $\mathrm{H}_{2}$ molecules under normal conditions, and so it is often regarded as diamagnetic.
} 


\subsubsection{Magnetism in solids}

Although on a fundamental level magnetism in solids is still governed by electron angular momentum and the presence of unpaired electrons, the origin of different magnetic states is a bit more complicated than for isolated atoms. However, the following discussion can be somewhat simplified by noting that in solids, the orbital angular momentum is to a large degree quenched due to the non-spherical symmetry of the potential, meaning that only the spin contribution to the total angular momenta of the electrons is important. This has the consequence that effects of spin-orbit coupling can be neglected when discussing phenomena such as finite-temperature magnetic transitions.

A central question with regards to solid state magnetism is the degree to which the electrons, and hence the (vector) magnetization density, defined as

$$
\boldsymbol{m}(\mathbf{r})=\sum_{i} \psi_{i}^{*}(\mathbf{r}) \boldsymbol{\sigma} \psi_{i}(\mathbf{r}),
$$

where the wavefunctions $\psi_{i}$ are solutions to the spin-dependent Kohn-Sham Hamiltonian and $\boldsymbol{\sigma}$ is the set of Pauli spin matrices [46], can be regarded as being spatially localized to a specific atom. In many metals, the conduction electrons are delocalized in the material - they are itinerant. The magnetic behavior of a metal can therefore often be described by the itinerant model of magnetism, first introduced by Stoner $[47,48]$. In this model, different magnetic states arise because of a redistribution of spin up and spin down states near the Fermi level, either due an externally applied magnetic field, as in Pauli paramagnetism (a weak form of paramagnetism) or because of exchange interactions between the electrons, as in weak ferromagnetism, the latter which is an example of an ordered form of magnetism where the macroscopic magnetization density is nonzero even in the absence of an external field.

However, for $3 d$ transition metals such as $\mathrm{Mn}, \mathrm{Fe}, \mathrm{Co}, \mathrm{Ni}$, the itinerant model is partially inadequate. For these metals, the itinerant model reproduces the $0 \mathrm{~K}$ ground state magnetic properties accurately, but fails to reproduce the behavior at finite temperatures [49]. For example, it significantly overestimates the critical magnetic order-disorder temperature $T_{c}$ - the temperature above which ordered magnetic states transition into paramagnetism - and results in a state of zero magnetization even on a local level just above this temperature, in conflict with experimental observations [49].

The magnetic properties of $3 d$ transition metals mainly arise from the $3 d$ electrons, which are itinerant only to a degree - they are still fairly strongly localized around the 
atomic nuclei. Hence, to accurately describe the finite temperature magnetic behavior of solids containing these metals, it is possible to adopt the local model of magnetism. In this model the magnetization density is strongly localized around the atomic nuclei, and in each point around a given nucleus oriented in approximately the same direction. In the interstitial regions, on the other hand, the magnetization density is low and, even though it can be oriented in complex noncollinear fashions, contributes only weakly to the total magnetic picture. This warrants the approximation that each atom possesses a distinct magnetic moment with a fixed magnitude and direction, just as if they were isolated atoms (the local model is also known as the atomic moment approximation) [46]. The local model provides an intuitively simple explanation of the magnetic state of a solid in terms of a set of specific spatial configurations of the atomic magnetic moments, which can be visualized as single vectors centered on the magnetic atoms in the lattice. As shown schematically in Fig. 6.1 (a), ferromagnetism can be explained under the local model by a parallel alignment of all atomic magnetic moments, caused by strong interatomic exchange interactions. Another form of ordered magnetism is antiferromagnetism, which can be explained by antiparallel atomic magnetic moments, as shown in Fig. 6.1 (b). The macroscopic magnetization of an antiferromagnetic solid is zero even in the presence of an external magnetic field.

The concept of local moments is also central to the disordered local moment model of paramagnetism (DLM), first introduced by Hubbard and Hasegawa, and further developed by Gyorffy et al. [49-51]. The idea behind the DLM is that the spatial orientations of the local magnetic moments become completely uncorrelated when the energies of the thermal fluctuations of the atoms considerably exceed the energies of the interatomic exchange interactions. Gyorffy et al. used the DLM to calculate the Curie temperature for $\mathrm{Fe}$, and showed that the magnitude of the magnetic moments remained significant above this temperature, in qualitative agreement with experiment [49].

In solids with negligble spin-orbit coupling, it is possible to consider only collinear magnetic configurations when carrying out DLM calculations, see Fig. 6.1 (c). This means that the problem becomes equivalent to that for calculations of a 50/50 binary alloy, and that it is therefore possible to use magnetically disordered supercells constructed using the SQS method. Another benefit is that collinear DFT calculations are significantly less computationally demanding than noncollinear calculations. 


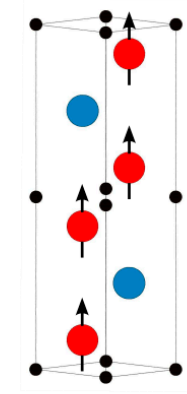

(a)

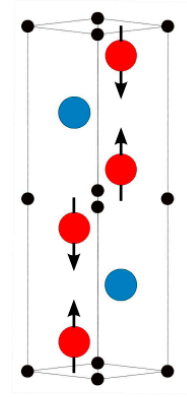

(b)

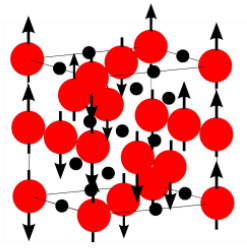

(c)

Figure 6.1. Schematic illustrations of different magnetic configurations under the local model of magnetism. (a) Ferromagnetism. (b) Antiferromagnetism. (c) DLM paramagnetism.

It should be noted that a more general theory of magnetism based on the concept of longitudinal and transverse spin fluctuations, in which the itinerant and local models constitute two extreme ends, has been developed by Moiyra [52]. According to this theory, itinerant magnetism arises due to strong temporal fluctuations in the magnitude of the local magnetization density, caused by the fast movement of the electrons in the solid. Localized magnetism, on the other hand, arises when only the transverse spin density fluctuations are important, which can be visualized as spatial rotations of the atomic moments.

\subsection{Magnetic energy and exchange interactions}

\subsubsection{The Heisenberg Hamiltonian}

Ground state properties of magnetic materials can be calculated using DFT, as it is straightforward to accommodate the underlying equations to spin polarized systems. However, for finite temperature properties such as the critical order-disorder temperature $T_{c}$, which is calculated for $\mathrm{Mn}_{2} \mathrm{GaC}$ in paper IV, the most practical approach is to combine DFT with statistical methods. A particularly common approach that has proven useful is to determine the exchange interactions $J_{i j}$ between pairs of magnetic atoms $i$ and $j$ in the lattice, and then let the sum of these parameters represent the magnetic Hamiltonian $H$, which, in the absence of an external magnetic field, is given by the Heisenberg Hamiltonian 


$$
H=-\sum_{i \neq j} J_{i j} \boldsymbol{e}_{i} \cdot \boldsymbol{e}_{j},
$$

where $\boldsymbol{e}_{i}$ and $\boldsymbol{e}_{j}$ are unit vectors parallel to the respective atomic magnetic moments. As in paper IV, Metropolis-type Monte Carlo simulations can be used to calculate the magnetic energy, magnetization, and specific heat, as well as other derived properties, at any temperature [53].

The Hamiltonian given by Eq. (6.2) contains only a bilinear term and is therefore a first order approximation of the deviation of the energy from that of the ideal DLM state; however, it is usually assumed that higher order approximations, which include terms such as the biquadratic and four-spin terms described in Ref. [54], are not necessary. Given this assumption, only collinear spin configurations need be considered when deriving the interactions, even if the interactions are later used in Monte Carlo simulations that allow for non-collinearity and entropy.

While there are systems in which the contributions from higher-order terms are still significant [55], this is likely not the case in $\mathrm{Mn}_{2} \mathrm{GaC}$, as it is shown in paper IV that the energy difference between PM (modeled using the DLM approach), and FM magnetic configurations from DFT calculations is reproduced by Monte Carlo simulations of Eq. (6.2).

\subsubsection{Magnetic exchange interactions}

The assumption of bilinear interactions, and thus the need for only collinear calculations, makes it possible to utilize methods for calculating exchange interaction parameters $J$ that were originally developed within the framework of binary alloy theory. Two such methods are direct cluster averaging (DCA), introduced by Berera et al. [56], and Connolly-Williams (CW) [57].

The magnetic direct cluster averaging method (MDCA), described in detail in paper IV, is a direct adaptation of DCA by Lindmaa et al., who used it to treat magnetism in chemically and topologically disordered phases [58]. In such phases, the signs and magnitudes of the exchange interactions may vary significantly even between pairs of atoms that would be equivalent in the ideal crystal lattice, due differences in the chemical environment or spatial separation. The benefit of the MDCA is that it gives direct access to interactions between any individual non-equivalent pair of atoms in the lattice. 
The CW method takes advantage of the symmetries present in ordered lattices by expressing the magnetic energy as

$$
H=-\sum_{\alpha} J_{\alpha} n_{\alpha} \Phi_{\alpha},
$$

where $n_{\alpha}$ is the number of atoms in coordination shell $\alpha$ with respect to atom $i$, and $\Phi_{\alpha}$ is the correlation function for this shell, defined for pair interactions as

$$
\Phi_{\alpha}=\frac{1}{n_{\alpha}} \sum_{j}^{n_{\alpha}} \varphi_{i} \varphi_{j} .
$$

In this equation, $\varphi_{i}$ and $\varphi_{j}$ can take on values of either +1 or -1 , depending on the directions of the atomic magnetic moments of atoms $i$ and $j$.

The $J_{\alpha}$ 's are determined by first calculating the ground state energies for several different magnetic configurations, including all correlation functions, and then solving the equation system

$$
\left.\begin{array}{cccccc}
H_{1} & H_{2} & \cdots & H_{N}
\end{array}\right)
$$

by, e.g. the linear least squares method. Due to the matrix inversion needed to solve for $J_{\alpha}$, the CW method is also known as the structure inversion method.

We conclude in paper IV that the methods give more or less equivalent results but that $\mathrm{CW}$ is better suited than MDCA for deriving J's in chemically and topologically ordered phases like $\mathrm{Mn}_{2} \mathrm{GaC}$, since the number of magnetic configurations needed from first-principles calculations are fewer using the former method, which significantly lessens the demand for computational resources.

\subsection{Magnetic MAX phases}

Although magnetic MAX phases have yet to find their way into any commercial products, their atomic-thin magnetic layers and strong interlayer exchange couplings make them potential candidates for magnetocaloric and magnetoelectric applications. 
The earliest reports on magnetic MAX phases were published a decade ago and were based entirely on theoretical calculations. They focused on $\mathrm{Cr}$ and $\mathrm{Fe}$ as the $\mathrm{M}$ element, as $\mathrm{Cr}$ is antiferromagnetic (AFM) in its elemental form, and Fe is ferromagnetic (FM).

Schneider et al. found the lowest energy configuration of $\mathrm{Cr}_{2} \mathrm{AlC}$ to be either paramagnetic (PM) or AFM (the electronic DOS and energy difference between the two configurations were very small), while the FM configuration was found to be metastable [59]. Dahlqvist et al. later calculated the enthalpy of several different AFM configurations of $\mathrm{Cr}_{2} \mathrm{AlC}$ and found that a particular AFM configuration denoted inAFM1 (for its in-plane antiferromagnetism) has the lowest enthalpy [45]. This also showed the importance of scanning the AFM configuration space. In another report, Zhou et al. concluded that the ground state of $\mathrm{Cr}_{2} \mathrm{GeC}$ is likely AFM [60].

In a report on the stability of Fe based MAX phases, Luo and Ahuja hypothesized, based on DFT calculations and comparisons with a limited number of competing phases, that it should be possible to synthesize $\mathrm{Fe}_{2} \mathrm{AlC}$ and $\mathrm{Fe}_{2} \mathrm{SiC}$ with AFM configurations [34]. However, in a subsequent report by Dahlqvist et al., it was shown that $\mathrm{Fe}_{2} \mathrm{AlC}$, when compared to all known competing phases, is not thermodynamically stable [61]. To date there have been no reports on the synthesis of any Fe-based MAX phases.

In contrast to the theoretical results, experimental evidence of magnetism in any $\mathrm{Cr}$ based MAX phases was lacking up until very recently. However, while it has now been established that both $\mathrm{Cr}_{2} \mathrm{AlC}$ and $\mathrm{Cr}_{2} \mathrm{GeC}$ as well as $\mathrm{Cr}_{2} \mathrm{GaC}$ and the nitride $\mathrm{Cr}_{2} \mathrm{GaN}$ are indeed magnetic, albeit with magnetic properties that are still not fully understood on a detailed level $[62,63]$, the very first experimentally verified magnetic MAX phase was the quaternary alloy $\left(\mathrm{Cr}_{0.75} \mathrm{Mn}_{0.25}\right)_{2} \mathrm{GeC}$. As reported by Ingason et al. in 2013 , this phase too was predicted from first-principles calculations, and it was successfully synthesized as a thin film through magnetron sputtering [64]. Vibrating sample magnetometry (VSM) measurements showed the phase to exhibit a magnetic response up to the maximum measurement temperature of $300 \mathrm{~K}$, although the exact magnetic configuration could not be completely determined. A small in-plane remanent magnetic moment suggested that the intralayer exchange coupling is at least partly ferromagnetic, but since the total remanence was significantly smaller than the saturation moment, and the saturation moment itself was very small compared to expectations based on the calculated moments, a purely FM configuration was ruled out. 
The synthesis of magnetic ( $\left.\mathrm{Cr}_{0.75} \mathrm{Mn}_{0.25}\right)_{2} \mathrm{GeC}$ was followed by the synthesis of two other Cr-Mn MAX phase alloys: ( $\left.\mathrm{Cr}_{1-x} \mathrm{Mn}_{x}\right)_{2} \mathrm{AlC}$ (in bulk and as a thin film), and ( $\mathrm{Cr}_{1-}$ $\left.{ }_{x} \mathrm{Mn}_{x}\right)_{2} \mathrm{GaC}$ (bulk), the former which had been predicted by Dahlqvist et al. a couple of years prior to the synthesis $[1,61,65,66]$. Just as for the Ge alloy, VSM measurements have shown that both phases exhibit a magnetic response up to at least room temperature, but their exact magnetic configurations have yet to be determined.

Particularly interesting about these MAX phase alloys is that the calculations suggest that the sign and magnitude of the exchange coupling between the Mn magnetic moments, and hence the magnetic configuration, can be tuned by altering the degree of chemical disorder on the $M$ sublattice. This could potentially be achieved by controlling the conditions during thin film synthesis [61].

Experimental verification of $\mathrm{Mn}_{2} \mathrm{GaC}$ the magnetic $\mathrm{MAX}$ phase investigated in this thesis was also reported by Ingason et al. in 2013 [67]. In fact, the preceding firstprinciples calculations of the stability of this phase, and its strong local Mn moments of around $1.5 \mu_{B}$, were two of the main reasons for the work on the Cr-Mn MAX alloys [61]. Currently $\mathrm{Mn}_{2} \mathrm{GaC}$ is probably the most well-explored MAX phase with respect to magnetic properties. In paper V (which is related to this thesis, but not included), theoretical and experimental methods have been used to probe the magnetic configuration as a function of temperature and magnetic field [68]. Using VSM measurements, an FM component was seen for temperatures up to about $250 \mathrm{~K}$, but just as for $\left(\mathrm{Cr}_{0.75} \mathrm{Mn}_{0.25}\right)_{2} \mathrm{GeC}$, the saturation moment was much greater than the remanent moment. There was further no easy axis, i.e., no spatial direction along which the Mn moments prefer to orient themselves, as determined by a comparison between out-of-plane and in-plane measurements of the magnetization. These two arguments therefore rule out a purely FM configuration. At temperatures from $250 \mathrm{~K}$ up to the maximum measurement temperature of $300 \mathrm{~K}$, no remanent moment could be observed, but a PM configuration was ruled out due to the presence of a magnetic signal.

A DFT based search for the ground state configuration indicates a particular antiferromagnetic configuration denoted AFM $[0001]_{4}^{A}$, schematically shown in Fig. 6.2 (a), in which the Mn atoms are parallel within the Mn layers but alternately parallel and antiparallel across the Ga layers. This configuration was replicated in Monte Carlo simulations of the bilinear Heisenberg Hamiltonian given by Eq. (6.2), constructed from exchange interaction parameters derived using the Connolly-Williams method applied to a large set of manually constructed, ordered magnetic configurations. For these configurations, the Mn atoms in each Mn-C-Mn trilayer were represented by a 
single supermoment, as a strong tendency for the Mn atoms to orient themselves in parallel across the $\mathrm{C}$ layers had been observed

The change in structural symmetry associated with the AFM $[0001]_{4}^{A}$ configuration, stemming from different spatial separations between the Mn layers that depend on the magnetic environment around the Ga layer, was shown to give rise to a characteristic peak at around $35^{\circ}$ in a simulated X-ray diffraction spectrum. An experimental spectrum obtained at $150 \mathrm{~K}$ displayed almost the same peak, thus supporting the results from the calculations. At room temperature, there was a match between simulated and experimental spectra of the $\mathrm{AFM}[0001]_{2}^{A}$ configuration, shown in Fig. 6.2 (b); partly for this reason the AFM[0001 $]_{2}^{A}$ geometry is used in paper IV when deriving the exchange interaction parameters for calculation of the critical orderdisorder temperature $T_{c}$, a quantity that was unknown prior to this work.

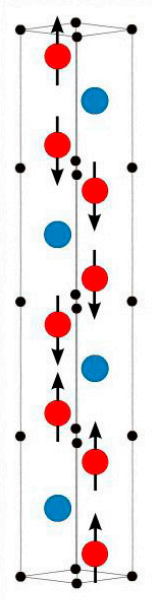

(a)

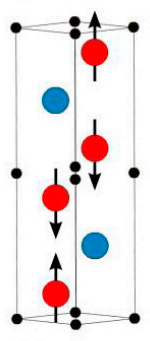

(b)

Figure 6.2 (a) The AFM[0001 $]_{4}^{A}$ antiferromagnetic configuration of $\mathrm{Mn}_{2} \mathrm{GaC}$. (b) $\mathrm{AFM}[0001]_{2}^{A}$. 


\section{Materials synthesis}

Nanolaminates such as MAX phases can be produced either through bulk or thin film synthesis.

\subsection{Bulk synthesis}

Bulk synthesis is a method in which a powder, consisting of solid grains generally between 0.1 and $100 \mu \mathrm{m}$ in size [69], are made to coalesce by adding enough heat and/or pressure allowing the atoms to diffuse across the grain boundaries.

In bulk synthesis, the system of particles strives to minimize its free energy, which depends both on the density of the resulting solid and the size, or coarseness, of the grains, as depicted in Fig. 7.1 (a)-(c). Densification leads to a decrease in the interfacial energy as the solid-vapor interfaces are replaced by lower-energy solid-solid interfaces. Grain coarsening, which occurs by means of Ostwald ripening and grain boundary migration, also leads to a decrease in the interfacial energy, since the number of grains and hence the total surface area of the system decreases.

(b)

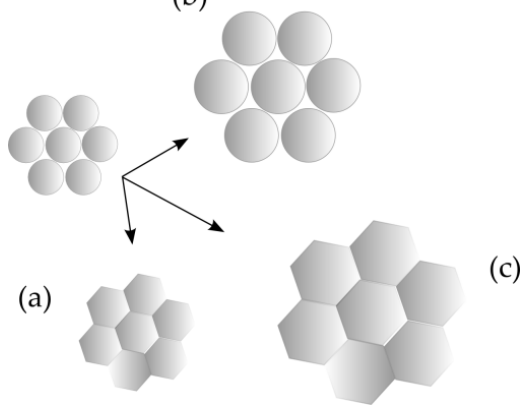

Figure 7.1. (a) Densification. (b) Coarsening. (c) Coarsening and densification.

In the initial stage of synthesis, densification is the dominant process, whereas both densification and grain coarsening play important roles in the intermediate and final stages. Since the densification rate is related to the grain size $G$ as $1 / G^{2}$, densification 
and grain coarsening are competing processes, with larger grains leading to slower densification [70].

The synthesis process and the microstructure of the resulting solid are affected by several different variables. Among these are material variables such as the average size and size distribution of the grains, the chemical composition, and the amount of impurities. There are also so-called process variables such as temperature, pressure, and atmosphere, i.e., the chemical environment in the sintering furnace. The MAX phase $\mathrm{Ti}_{2} \mathrm{AlC}$, which is one of the phases discussed in paper $\mathrm{I}$, has been synthesized using many different combinations of material and process variables. It has, for example, been synthesized using both hot pressing (HP) and hot isostatic pressing (HIP), two techniques characterized by high temperatures and pressures (uniaxial and uniform, respectively). Both HP and HIP create highly dense and phase pure $\mathrm{Ti}_{2} \mathrm{AlC}$ with excellent mechanical properties [71].

\subsection{Thin film synthesis}

In thin film synthesis, atoms are deposited onto the surface of a substrate material to form a film which can range in thickness from a single atomic layer of a few Ångströms to a layer of several micrometers. Growth of thin films can be either epitaxial, where the crystal structure is oriented in such a way that it follows a particular crystallographic orientation of the substrate, or non-epitaxial, where the film is randomly oriented with respect to the substrate. Epitaxial growth is beneficial as it offers improved control of the microstructure and hence the physical properties of the film.

Just as for bulk synthesis, the driving force for thin film growth is to reduce the free energy of the system. The thin film growth can proceed in three different modes, depending on the relative magnitudes of the film-surface, film-vapor, and substratevapor energies $^{10}$ : i) Volmer-Weber (VW) growth, ii) Frank-van der Merwe (FM) growth, and iii) Stranski-Krastanov (SK) growth.

In VW growth (Fig $7.2(\mathrm{a})$ ), the sum of the film-substrate and film-vapor energies is greater than the substrate-vapor energy, which leads to clustering of the adatoms into $3 \mathrm{D}$ islands that grow in size and eventually coalesce to form a continuous film. This growth mode is obtained when there is a large lattice mismatch, and hence large strain,

\footnotetext{
${ }^{10}$ Note that these are specific surface energies, i.e., energies per unit surface.
} 
between the film and the substrate, either due to different lattice parameters, or different crystal structure.

In FM growth (Fig 7.2 (b)), also known as layer-by-layer growth, the sum of the filmsubstrate and film-vapor energies is instead smaller than the substrate-vapor energy, thus promoting the formation and eventual coalescence of 2D islands, i.e., islands only one atom thick. This occurs when the film and the substrate have matching structures and identical, or close to identical, lattice parameters.

SK growth (Fig 7.2 (c)), finally, is a combination of VW and FM growth modes, where $2 \mathrm{D}$ layer formation is followed by growth and coalescence of $3 \mathrm{D}$ islands on the top layer.

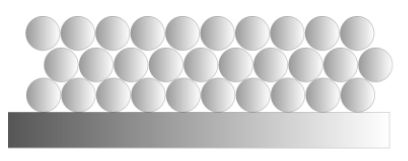

(a)

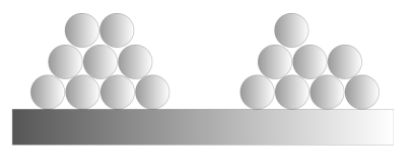

(b)

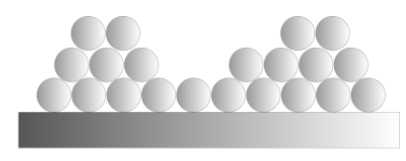

(c)

Figure 7.2 (a) Volmer-Weber growth. (b) Frank-van der Merwe. (c) Stranski-Krastanov.

Methods for achieving thin film growth are usually categorized as either chemical vapor deposition (CVD), or physical vapor deposition (PVD). In CVD, a gaseous mix of volatile precursor molecules at least partly made up of the atoms intended to form the film are introduced into a vacuum chamber. The precursors then condense onto a substrate, leaving the film-forming atoms on the substrate surface.

While it is possible to achieve MAX phase thin film growth using CVD techniques, it is more common to use PVD techniques, for example magnetron sputtering. In magnetron sputtering, chemically inert atoms such as argon are first ionized in a vacuum chamber, and then accelerated towards elemental or compound targets. The impact leads to ejection, or sputtering, of target particles, which then travel to the substrate, onto which they condense. One of the investigated phases in this thesis, $\mathrm{Mn}_{2} \mathrm{GaC}$ has so far only been produced using this technique. 


\subsection{Differences between bulk and thin film synthesis}

Perhaps the most important difference between bulk and thin film synthesis is that the former is primarily an equilibrium process and is therefore restricted by the phase diagram of the investigated materials system, whereas the latter allows for the formation of metastable phases. This can be achieved by facilitated diffusion of the adatoms through, for instance, ion bombardment [72], which means that thin film synthesis temperatures are typically lower than the temperatures needed for bulk synthesis.

Another difference is the attainable crystal quality from bulk versus thin film synthesis, where the former typically results in polycrystalline samples of random orientation, while the latter more easily allows for control of crystal orientation through epitaxy. For a given solid phase of a given crystal quality, the physical properties may therefore vary depending on the synthesis method. An example that illustrates this is the electrical resistivity of $\mathrm{Ti}_{2} \mathrm{AlC}$, which in a thin film of this phase has been measured to $0.36 \mu \Omega \cdot \mathrm{m}$ at room temperature, but to $0.65 \mu \Omega \cdot \mathrm{m}$ in a bulk sample [73]. 


\section{Summary of included papers}

\subsection{Paper I: Temperature dependent phase stability of nanolaminated ternaries from first-principles calculations}

In this paper, we investigate the phase stability as a function of temperature of three MAX phases: $\mathrm{Ti}_{2} \mathrm{AlC}$, $\mathrm{Ti}_{3} \mathrm{AlC}_{2}$, and $\mathrm{Ti}_{4} \mathrm{AlC}_{3}$. We compare the results to previous predictions from $0 \mathrm{~K}$ calculations, in order to determine the importance of the inclusion of temperature dependent effects with respect to studies of MAX phase stability.

The Gibbs free energy of the three MAX phases as well as of 14 competing phases within the Ti-Al-C system was calculated using density functional theory and density functional perturbation theory, both as implemented in VASP. The temperature dependent effects included were the electronic free energy, the vibrational free energy, the configurational free energy, and thermal expansion; however, results from calculations both including and excluding thermal expansion were considered and compared.

The results show that for a temperature of up to $2000 \mathrm{~K}$, the Gibbs free energy of formation for each MAX phase is essentially the same as obtained at $0 \mathrm{~K}$, thus meaning that the phase stability is only weakly dependent on temperature. The reason for this is twofold: first, each individual temperature dependent contribution to the Gibbs free energy for each MAX phase is to a large extent cancelled by the corresponding contribution for their respective sets of most competing phases. Second, the individual contributions to the Gibbs free energy of formation partially cancel each other.

Our results suggest that phase stability of MAX phases is mainly governed by the zerotemperature energy term, and that to go beyond such calculations for phase stability predictions is therefore not motivated, with the exception of borderline cases in zerotemperature investigations. 


\subsection{Paper II: Phase stability of the nanolaminates $\mathrm{V}_{2} \mathrm{Ga}_{2} \mathrm{C}$ and $\left(\mathrm{Mo1}_{-x} \mathrm{~V}_{x}\right)_{2} \mathrm{Ga}_{2} \mathrm{C}$ from first-principles calculations}

Here we investigate, using first-principles calculations and a linear optimization procedure, the phase stability, electronic, and elastic properties of $\mathrm{V}_{2} \mathrm{Ga}_{2} \mathrm{C}$, and the phase stability of the alloy $\left(\mathrm{Mo1}_{-x} \mathrm{~V}_{x}\right)_{2} \mathrm{Ga}_{2} \mathrm{C}$. Both phases are believed to belong to a suggested new family of materials closely related to the MAX phase family, due to their hexagonal, layered structures. The alloy is especially interesting as it holds promise as a material from which $\left(\mathrm{Mo}_{1-x} \mathrm{~V}_{x}\right)_{2} \mathrm{C}$, a MXene, can be synthesized.

For $\mathrm{V}_{2} \mathrm{Ga}_{2} \mathrm{C}$, the Gibbs free energy of formation is determined to be $-0.6 \mathrm{meV} /$ atom, and out of all possible competing phases in the $\mathrm{V}-\mathrm{Ga}-\mathrm{C}$ phase diagram, the set of most competing phases is identified to be $\mathrm{V}_{3} \mathrm{Ga}_{2} \mathrm{C}_{2}, \mathrm{~V}_{2} \mathrm{GaC}$, and $\mathrm{V}_{8} \mathrm{Ga}_{41}$. This indicates that $\mathrm{V}_{2} \mathrm{Ga}_{2} \mathrm{C}$ is possible to synthesize. Further, the electronic band structure exhibits considerable anisotropy, and from the five elastic constants $C_{11}, C_{12}, C_{13}, C_{33}$, and $C_{44}$, we determine the bulk, shear, and Young's modulus to be 141, 95, and $232 \mathrm{GPa}$, respectively. We further determine Poisson's ratio to be 0.23 .

For $\left(\mathrm{Mo1}-x \mathrm{~V}_{x}\right)_{2} \mathrm{Ga}_{2} \mathrm{C}$, we calculate the Gibbs free energy of formation for three different vanadium concentrations: $x=0.25,0.5$, and 0.75 . A thorough scan of the Mo-V-Ga-C phase diagram has been performed to identify the set of most competing phases at each concentration. Around $x=0.25$, we predict the stability of both an ordered and a disordered solid solution on the $M$ sublattice, the latter which has been modeled using the SQS approach. The ordered solution is suggested to be stable below $1000 \mathrm{~K}$, whereas the disordered one is stabilized when the entropic contribution to the Gibbs free energy becomes significant, which happens above $1000 \mathrm{~K}$. For $\mathrm{x}=0.5$, only a disordered solutions is stable, above $2100 \mathrm{~K}$. Lastly, for $\mathrm{x}=0.75$, stability is indicated at $1750 \mathrm{~K}$. 


\subsection{Paper III: First-principles calculations of the electronic, vibrational, and electronic properties of the magnetic laminate $\mathrm{Mn}_{2} \mathrm{GaC}$}

In this paper, selected properties of one of the first magnetic MAX phases to be synthesized, $\mathrm{Mn}_{2} \mathrm{GaC}$, are studied.

The electronic properties have been investigated by evaluating the electronic band structure and electronic density of states. The energy bands cross the Fermi level in the horizontal directions in reciprocal space, but not in the vertical directions, indicating that electrical conduction in $\mathrm{Mn}_{2} \mathrm{GaC}$ might be anisotropic, occurring mostly in-plane.

We have further investigated the elastic properties, which were derived from the five elastic constants $C_{11}, C_{12}, C_{13}, C_{33}$, and $C_{44}$. Evaluated elastic properties were compared to theoretical and experimental results for $M_{2} A C$ phases where $M=\mathrm{Ti}, \mathrm{V}, \mathrm{Cr}, \mathrm{Zr}, \mathrm{Nb}$, Ta, and $A=\mathrm{Al}, \mathrm{S}, \mathrm{Ge}, \mathrm{In}, \mathrm{Sn}$. The Voigt bulk modulus was determined to be $157 \mathrm{GPa}$, the Voigt shear modulus $93 \mathrm{GPa}$, the Young's modulus $233 \mathrm{GPa}$, and the Poisson's ratio 0.25 . As compared to other $M_{2} A C$ phases, the bulk and shear moduli are concluded to be fairly low, whereas the Young's modulus is intermediate, and the Poisson's ratio high. Furthermore, according to theoretical criteria for elastic isotropy based on the elastic constants, $\mathrm{Mn}_{2} \mathrm{GaC}$ was found relatively elastically isotropic, with a compression anisotropy factor of 0.97 , and shear anisotropy factors of 0.9 and 1 , respectively. Two machinability indices were also calculated, which indicate that $\mathrm{Mn}_{2} \mathrm{GaC}$ is machinable.

For all properties here investigated, the choice of magnetic configuration has a nonnegligible effect. This underlines the importance of identifying the most relevant magnetic spin configuration, i.e. the ground state for low temperatures and disordered paramagnetic states at higher temperatures, before evaluating physical MAX phase properties. 


\subsection{Paper IV: Magnetic exchange interactions and critical temperature of the nanolaminate $\mathrm{Mn}_{2} \mathrm{GaC}$}

In this work, we employ and critically evaluate a first-principles-based supercell approach for predicting the magnetic critical order-disorder temperature $T_{c}$. Supercell calculations have the benefit of allowing for straightforward incorporation of structural or vibrational disorder effects on the magnetic interactions. As a model material we use the recently discovered nanolaminate $\mathrm{Mn}_{2} \mathrm{GaC}$.

First, we derive the pair exchange interaction parameters $J_{i j}$ of the bilinear Heisenberg Hamiltonian using the novel magnetic direct cluster averaging method (MDCA). In this method, the $J$ 's are calculated through an averaging procedure over a large number of supercells in which the magnetic state has been randomly generated with respect to all magnetic atoms except for those of atoms $i$ and $j$, for which $J_{i j}$ is sought. We compare the J's from the MDCA calculations to the same parameters calculated using the Connolly-Williams (CW) method, and show that the two methods yield closely matching results; however, we conclude that for highly orderd phases such as $\mathrm{Mn}_{2} \mathrm{GaC}, \mathrm{CW}$ may be preferred due to a significant reduction of the required computational resources.

Secondly, Monte Carlo simulations are used to derive the magnetic energy, total magnetic moment, specific heat, magnetic susceptibility, as well as $T_{c}$. For $\mathrm{Mn}_{2} \mathrm{GaC}$, we find that $T_{c}=660 \mathrm{~K}$. The uncertainty in the calculated value of $T_{c}$ caused by uncertainties in the MDCA-derived $J$ 's is discussed and exemplified in our case by a calculation of the standard deviation, which is $133 \mathrm{~K}$. The value of $T_{c}$ remains to be experimentally verified. 


\section{Contribution to the field}

The work presented in this thesis is based solely on theoretical methods, and it consists to a large extent of predictions, most of which have yet to be experimentally verified. Upon future experimental work, these predictions may provide valuable insights into the accuracy of the chosen methods.

The most important predictions that have been made are the following:

- that it is possible to synthesize the MAX-like phase $\mathrm{V}_{2} \mathrm{Ga}_{2} \mathrm{C}$

- that it has a bulk, shear, and Young's modulus of approximately 141, 95, and $232 \mathrm{GPa}$, respectively

- that it is possible to synthesize the MAX-like alloy $\left(\mathrm{Mo1-x} \mathrm{V}_{x}\right)_{2} \mathrm{GaC}$

- that $\mathrm{Mn}_{2} \mathrm{GaC}$ has a magnetic critical order-disorder temperature that is well above room temperature

- that its bulk, shear, and Young's modulus are approximately 157, 92, and 233 $\mathrm{GPa}$, respectively

- that the remarkable accuracy of previous MAX phase stability predictions at $0 \mathrm{~K}$ conditions can be explained by cancellation of temperature dependent contributions to the Gibbs free energy

Additionally, we have compared two different methods for calculating exchange interaction parameters in magnetic materials: the Connolly-Williams method (CW) and the magnetic direct cluster averaging method (MDCA). We conclude that

- $\mathrm{CW}$ is the better choice for highly ordered phases

- MDCA is the better choice for disordered phases

This is valuable information for researchers investigating magnetism from a theoretical perspective. 


\section{References}

[1] A. Mockute, M. Dahlqvist, J. Emmerlich, L. Hultman, J.M. Schneider, P.O.Å. Persson, J. Rosen, Physical Review B, 87 (2013) 094113.

[2] S. Dubois, G.P. Bei, C. Tromas, V. Gauthier-Brunet, P. Gadaud, International Journal of Applied Ceramic Technology, 7 (2010) 719-729.

[3] M.W. Barsoum, T. El-Raghy, M. Ali, Metall and Mat Trans A, 31 (2000) 1857-1865.

[4] P. Eklund, M. Beckers, U. Jansson, H. Högberg, L. Hultman, Thin Solid Films, 518 (2010) 18511878.

[5] C. Hu, C.C. Lai, Q. Tao, J. Lu, J. Halim, L. Sun, J. Zhang, J. Yang, B. Anasori, J. Wang, Y. Sakka, L. Hultman, P. Eklund, J. Rosen, M.W. Barsoum, Chemical Communications, 51 (2015) 6560-6563.

[6] C.C. Lai, R. Meshkian, M. Dahlqvist, J. Lu, L.Å. Näslund, O. Rivin, E.N. Caspi, O. Ozeri, L. Hultman, P. Eklund, M.W. Barsoum, J. Rosen, Acta Materialia, 99 (2015) 157-164.

[7] M. Naguib, V.N. Mochalin, M.W. Barsoum, Y. Gogotsi, Advanced Materials, 26 (2014) 992-1005.

[8] W. Jeitschko, H. Nowotny, F. Benesovsky, Journal of the Less Common Metals, 7 (1964) 133138.

[9] W. Jeitschko, H. Nowotny, Monatshefte für Chemie, 98 (1967) 329-337.

[10] H. Wolfsgruber, H. Nowotny, F. Benesovsky, Monatshefte für Chemie, 98 (1967) 2403-2405.

[11] M.W. Barsoum, T. El-Raghy, American Scientist, 89 (2001) 334-343.

[12] M.W. Barsoum, T. El-Raghy, Journal of the American Ceramic Society, 79 (1996) 1953-1956.

[13] M.W. Barsoum, M. Radovic, American Ceramic Society Bulletin, 92 (2013) 28.

[14] M. Catlin, in, Google Patents, 2005.

[15] S\&S Cycle Inc., in, S\&S Cycle, Inc., http://www.sscycle.com/.

[16] Laser Design Inc, in, Laser Design Inc, http://laserdesign.com/.

[17] SANDVIK Coromant, in, SANDVIK Coromant, http://www.sandvik.coromant.com/enus/pages/default.aspx?country=us.

[18] J. Frodelius, E.M. Johansson, J.M. Córdoba, M. Odén, P. Eklund, L. Hultman, International Journal of Applied Ceramic Technology, 8 (2011) 74-84.

[19] D.J. Tallman, B. Anasori, M.W. Barsoum, Materials Research Letters, 1 (2013) 115-125.

[20] L.E. Toth, Journal of the Less Common Metals, 13 (1967) 129-131.

[21] R. Meshkian, A.S. Ingason, M. Dahlqvist, A. Petruhins, U.B. Arnalds, F. Magnus, J. Lu, J. Rosen, physica status solidi (RRL) - Rapid Research Letters, 9 (2015) 197-201.

[22] P. Hohenberg, W. Kohn, Physical Review, 136 (1964) B864-B871.

[23] W. Kohn, L.J. Sham, Physical Review, 140 (1965) A1133-A1138.

[24] K. Burke, J.P. Perdew, M. Ernzerhof, The Journal of Chemical Physics, 109 (1998) 3760-3771. 
[25] C.S. Wang, B.M. Klein, H. Krakauer, Physical Review Letters, 54 (1985) 1852-1855.

[26] J. Kübler, Solid State Communications, 72 (1989) 631-633.

[27] F.O. Kannemann, A.D. Becke, Journal of Chemical Theory and Computation, 5 (2009) 719-727.

[28] J.P. Perdew, K. Burke, M. Ernzerhof, Physical Review Letters, 77 (1996) 3865-3868.

[29] A.E. Mattsson, P.A. Schultz, M.P. Desjarlais, T.R. Mattsson, K. Leung, Modelling and Simulation in Materials Science and Engineering, 13 (2005) R1.

[30] P.E. Blöchl, Physical Review B, 50 (1994) 17953-17979.

[31] G. Trimarchi, A. Zunger, Physical Review B, 75 (2007) 104113.

[32] J.P. Palmquist, S. Li, P.O.Å. Persson, J. Emmerlich, O. Wilhelmsson, H. Högberg, M.I. Katsnelson, B. Johansson, R. Ahuja, O. Eriksson, L. Hultman, U. Jansson, Physical Review B, 70 (2004) 165401. [33] V.J. Keast, S. Harris, D.K. Smith, Physical Review B, 80 (2009) 214113.

[34] W. Luo, R. Ahuja, Journal of Physics: Condensed Matter, 20 (2008) 064217.

[35] M. Dahlqvist, B. Alling, J. Rosén, Physical Review B, 81 (2010) 220102.

[36] R. Frederick, Fundamentals of Statistical and Thermal Physics, Waveland Press, Inc., 1965.

[37] X. Gonze, C. Lee, Physical Review B, 55 (1997) 10355-10368.

[38] S. Baroni, S. de Gironcoli, A. Dal Corso, P. Giannozzi, Reviews of Modern Physics, 73 (2001) 515-562.

[39] A. Zunger, S.H. Wei, L.G. Ferreira, J.E. Bernard, Physical Review Letters, 65 (1990) 353-356.

[40] A. van de Walle, G. Ceder, Reviews of Modern Physics, 74 (2002) 11-45.

[41] A. Togo, L. Chaput, I. Tanaka, G. Hug, Physical Review B, 81 (2010) 174301.

[42] M.F. Cover, O. Warschkow, M.M.M. Bilek, D.R. McKenzie, Journal of Physics: Condensed Matter, 21 (2009) 305403.

[43] M.F. Cover, O. Warschkow, M.M.M. Bilek, D.R. McKenzie, Advanced Engineering Materials, 10 (2008) 935-938.

[44] L. Fast, J.M. Wills, B. Johansson, O. Eriksson, Physical Review B, 51 (1995) 17431-17438.

[45] M. Dahlqvist, B. Alling, J. Rosén, Journal of Applied Physics, 113 (2013) 216103.

[46] E. Sjöstedt, L. Nordström, Physical Review B, 66 (2002) 014447.

[47] E.C. Stoner, Proceedings of the Royal Society of London A: Mathematical, Physical and Engineering Sciences, 154 (1936) 656-678.

[48] E.C. Stoner, Proceedings of the Royal Society of London A: Mathematical, Physical and Engineering Sciences, 165 (1938) 372-414.

[49] B.L. Gyorffy, A.J. Pindor, J. Staunton, G.M. Stocks, H. Winter, Journal of Physics F: Metal Physics, 15 (1985) 1337.

[50] H. Hasegawa, Journal of the Physical Society of Japan, 46 (1979) 1504-1514.

[51] J. Hubbard, Physical Review B, 19 (1979) 2626-2636.

[52] T. Moriya, Journal of Magnetism and Magnetic Materials, 14 (1979) 1-46. 
[53] N. Metropolis, A.W. Rosenbluth, M.N. Rosenbluth, A.H. Teller, E. Teller, The Journal of Chemical Physics, 21 (1953) 1087-1092.

[54] R. Drautz, M. Fähnle, Physical Review B, 72 (2005) 212405.

[55] N.S. Fedorova, C. Ederer, N.A. Spaldin, A. Scaramucci, Physical Review B, 91 (2015) 165122.

[56] A. Berera, H. Dreysse, L.T. Willes, D.d. Fontaine, Journal of Physics F: Metal Physics, 18 (1988) L49.

[57] J.W.D. Connolly, A.R. Williams, Physical Review B, 27 (1983) 5169-5172.

[58] A. Lindmaa, R. Lizárraga, E. Holmström, I.A. Abrikosov, B. Alling, Physical Review B, 88 (2013) 054414.

[59] J.M. Schneider, Z. Sun, R. Mertens, F. Uestel, R. Ahuja, Solid State Communications, 130 (2004) 445-449.

[60] W. Zhou, L. Liu, P. Wu, Journal of Applied Physics, 106 (2009) 033501.

[61] M. Dahlqvist, B. Alling, I.A. Abrikosov, J. Rosen, Physical Review B, 84 (2011) 220403.

[62] M. Jaouen, M. Bugnet, N. Jaouen, P. Ohresser, V. Mauchamp, T. Cabioc'h, A. Rogalev, Journal of Physics: Condensed Matter, 26 (2014) 176002.

[63] Z. Liu, T. Waki, Y. Tabata, K. Yuge, H. Nakamura, I. Watanabe, Physical Review B, 88 (2013) 134401.

[64] A.S. Ingason, A. Mockute, M. Dahlqvist, F. Magnus, S. Olafsson, U.B. Arnalds, B. Alling, I.A. Abrikosov, B. Hjörvarsson, P.O.Å. Persson, J. Rosen, Physical Review Letters, 110 (2013) 195502. [65] A. Mockute, J. Lu, E.J. Moon, M. Yan, B. Anasori, S.J. May, M.W. Barsoum, J. Rosen, Materials Research Letters, (2014) 1-7.

[66] A. Mockute, P.O.Å. Persson, F. Magnus, A.S. Ingason, S. Olafsson, L. Hultman, J. Rosen, physica status solidi (RRL) - Rapid Research Letters, 8 (2014) 420-423.

[67] A.S. Ingason, A. Petruhins, M. Dahlqvist, F. Magnus, A. Mockute, B. Alling, L. Hultman, I.A. Abrikosov, P.O.Å. Persson, J. Rosen, Materials Research Letters, 2 (2014) 89-93.

[68] M. Dahlqvist, A.S. Ingason, B. Alling, F. Magnus, A. Thore, A. Petruhins, A. Mockute, R. Meshkian, M. Sahlberg, P.O.Å. Persson, B. Hjörvarsson, I.A. Abrikosov, J. Rosén, (To be submitted.), (2014).

[69] S.-J.L. Kang, 2 - Thermodynamics of The Interface2, in: S.-J.L. Kang (Ed.) Sintering, Butterworth-Heinemann, Oxford, 2005, pp. 9-18.

[70] N.P. Bansal, A.R. Boccaccini, in, John Wiley \& Sons, New Jersey, 2012.

[71] X.H. Wang, Y.C. Zhou, Journal of Materials Science \& Technology, 26 (2010) 385-416.

[72] R. Ditchfield, E.G. Seebauer, Physical Review B, 63 (2001) 125317.

[73] V. Mauchamp, W. Yu, L. Gence, L. Piraux, T. Cabioc'h, V. Gauthier, P. Eklund, S. Dubois, Physical Review B, 87 (2013) 235105. 


\section{Papers}

The articles associated with this thesis have been removed for copyright reasons. For more details about these see:

http://urn.kb.se/resolve?urn=urn:nbn:se:liu:diva-124564 